\title{
The kinematics and magnetic fields in water-fountain sources based on $\mathrm{OH}$ maser observations ${ }^{\star}$
}

\author{
N. Amiri ${ }^{1,3}$, W. Vlemmings ${ }^{2}$, and H. J. van Langevelde ${ }^{3,1}$ \\ ${ }^{1}$ Sterrewacht Leiden, Leiden University, Niels Bohrweg 2, 2333 CA Leiden, The Netherlands \\ e-mail: amiri@strw.leidenuniv.n \\ 2 Argelander Institute for Astronomy, University of Bonn, Auf dem Hügel 71, 53121 Bonn, Germany \\ 3 Joint Institute for VLBI in Europe (JIVE), Postbus 2, 7990 AA Dwingeloo, The Netherlands
}

Received 4 February 2011 / Accepted 21 June 2011

\section{ABSTRACT}

\begin{abstract}
Context. water-fountain sources are a class of post-AGB objects that exhibit bipolar jet-like structures traced by $\mathrm{H}_{2} \mathrm{O}$ maser emission. The circumstellar envelopes of these objects show deviations from spherical symmetry. While the $\mathrm{H}_{2} \mathrm{O}$ masers originate in a collimated outflow in the polar region, the $\mathrm{OH}$ masers may be distributed in the equatorial region or a biconical outflow in the circumstellar shell. Magnetic fields could play an important role in collimating the jet and shaping the circumstellar envelope of these objects. Aims. The aim of this project is to understand the morphology of the $\mathrm{OH}$ masers in three water-fountain sources, for which we obtained high-resolution interferometric data. We compare the observational parameters of the spectral profile and spatial distribution with our models in order to constrain the morphology and velocity fields of the $\mathrm{OH}$ maser shell. We would also like to understand the role of the magnetic field in shaping the circumstellar envelope of these stars.

Methods. We observed the $\mathrm{OH}$ masers of three water-fountain sources $(\mathrm{OH} 12.8-0.9, \mathrm{OH} 37.1-0.8$, and W43A) in full polarization spectral line mode using the UK Multi-Element Radio Linked Interferometer Network. Additionally, we performed reconstruction models of $\mathrm{OH}$ maser shells distributed uniformly in equatorial or biconical outflows and compared them with the $\mathrm{OH}$ maser observations.

Results. The $\mathrm{OH}$ masers of W43A seem to be located in the equatorial region of the circumstellar shell, while for $\mathrm{OH} 12.8-0.9$ the masers are likely distributed in a biconical outflow. We measured magnetic fields of $\sim 360 \mu \mathrm{G}$ and $\sim 29 \mu \mathrm{G}$ on average for $\mathrm{OH} 37.1-0.8$ and $\mathrm{OH} 12.8-0.9$, respectively. The measured field strengths show that a large-scale magnetic field is present in the circumstellar environment of these stars.

Conclusions. Our observations show that the $\mathrm{OH}$ maser region of the three water-fountain sources studied in this work show signs of aspherical expansion. Reconstruction of the $\mathrm{OH}$ maser distribution of these objects show both biconical and equatorial distributions. Magnetic fields likely play an important role in shaping the $\mathrm{OH}$ maser region of water-fountain sources.
\end{abstract}

Key words. stars: individual: W43A - magnetic fields - polarization - masers - stars: individual: OH 12, 8-0.9 stars: individual: $\mathrm{OH} 37,1-0.8$

\section{Introduction}

Maser emission occurs in different regions of the circumstellar envelope (CSE) of evolved stars and can be studied at high angular resolution. These masers are good tracers of the outflow and morphology. In the picture of regular asymptotic giant branch (AGB) stars, $\mathrm{SiO}$ masers occur close to the central star, while the $\mathrm{H}_{2} \mathrm{O}$ and $\mathrm{OH}$ masers are found at progressively further distances (e.g. Habing 1996). The OH masers often exhibit double-peak profiles, while the $\mathrm{H}_{2} \mathrm{O}$ maser spectra are typically more irregular and have a velocity range confined within the $\mathrm{OH}$ spectrum (e.g. Reid \& Moran 1981).

Water-fountain sources are defined by having high-velocity $\mathrm{H}_{2} \mathrm{O}$ masers, and they are observed to have jets. The spectral characteristic and the spatial morphology of the $\mathrm{OH}$ and $\mathrm{H}_{2} \mathrm{O}$ masers of these objects are unique and differ from those of typical AGB stars. The $\mathrm{OH}$ and $\mathrm{H}_{2} \mathrm{O}$ masers of these objects show double-peak profiles; however, the $\mathrm{H}_{2} \mathrm{O}$ maser velocity range exceeds that of $\mathrm{OH}$ masers (Likkel et al. 1992). For example, OH 09.1-0.4 has water maser emission spread over nearly

* Figures 6-19 are available in electronic form at http://www . aanda.org
$400 \mathrm{~km} \mathrm{~s}^{-1}$ (Walsh et al. 2009). Therefore, these objects can not be understood by the standard expanding shell model. An archetype of this class of objects is W43A, for which interferometric observations showed highly collimated $\mathrm{H}_{2} \mathrm{O}$ maser jets, with a 3D outflow velocity of $145 \mathrm{~km} \mathrm{~s}^{-1}$ (Imai et al. 2002). Interferometric observations of the $\mathrm{H}_{2} \mathrm{O}$ masers of other water-fountains (e.g. IRAS 16342-3814, IRAS 19134+2131, and $\mathrm{OH}$ 12.8-0.9) also revealed bipolar distributions (Morris et al. 2003; Claussen et al. 2004; Boboltz \& Marvel 2005).

The bipolar jets observed in water-fountain sources are presumably to be related to the onset of asymmetric planetary nebulae (PNe) (Sahai \& Trauger 1998). The majority of the observed planetary nebulae show elliptical or bipolar structures (Manchado et al. 2000). During the post-AGB phase, the jet-like outflows modify the CSEs, for example, by carving out polar cavities. It could be this imprint that provides the morphological signature of the aspherical PNe. These jets are thought to form on a very short time scale, less than 100 years during the transition from the AGB phase to PNe (Imai et al. 2002).

Although $\mathrm{H}_{2} \mathrm{O}$ masers are found in bipolar jet-like structures in water-fountain sources, the distribution of $\mathrm{OH}$ masers in the CSE of these stars is not clear. For example, the blue-shifted 
and red-shifted $\mathrm{OH}$ masers of $\mathrm{W} 43 \mathrm{~A}$ are separated in the plane of the sky and in the opposite direction with respect to the blueshifted and red-shifted $\mathrm{H}_{2} \mathrm{O}$ masers (Diamond et al. 1985; Likkel et al. 1992; Imai et al. 2002). Likkel et al. (1992) proposed that the $\mathrm{OH}$ masers of W43A are located in a biconical outflow surrounding the $\mathrm{H}_{2} \mathrm{O}$ maser jet at lower latitudes. Likewise, Sahai et al. (1999) suggest that the $\mathrm{OH}$ masers of the water-fountain source IRAS 16342-3814 are located at intermediate systemic latitudes, where the outflow velocity is much lower than along the polar axis. So far, no systematic studies have been performed to explain the morphology of the $\mathrm{OH}$ masers in water-fountains. Therefore, it is important to investigate observational parameters, such as the profile shape and spatial distribution, and compare them with geometrical models to constrain the morphology and velocity field of these objects.

The $\mathrm{H}_{2} \mathrm{O}$ maser jets observed in water-fountain sources may result from magnetic collimation (García-Segura et al. 2005). Theoretical models indicated that magnetic fields in AGB stars are driven by a dynamo. However, in order to maintain the differential rotation an additional source of angular momentum such as a binary companion or a heavy planet is required (Nordhaus et al. 2007). In addition to probing the morphology of the CSEs, masers are useful tracers of the magnetic field strength and structure around these stars. For example, $\mathrm{H}_{2} \mathrm{O}$ maser polarimetric observations of W43A showed that the jet is magnetically collimated (Vlemmings et al. 2006). Follow up polarimetric observations of the $\mathrm{OH}$ maser region showed evidence for a large scale magnetic field in the circumstellar environment of this star (Amiri et al. 2010, hereafter A10).

In this paper, we present the $1612 \mathrm{MHz} \mathrm{OH}$ maser polarimetric observations of three water-fountain candidates (W43A, $\mathrm{OH}$ 12.8-0.9 and $\mathrm{OH} 37.1-0.8)$. For two sources, $\mathrm{OH}$ 12.8-0.9 \& OH 37.1-0.8 the observations are presented for the first time. The observations are compared with the kinematical reconstruction models in order to understand the $\mathrm{OH}$ maser morphology of these objects. In Sect. 2 we describe the MERLIN observations. The results of the observations are shown in Sect. 3. The modeling procedure and comparison with observations are discussed in Sect. 4.

\section{MERLIN observations}

We observed the $1612 \mathrm{MHz} \mathrm{OH}$ masers of three waterfountain sources with the UK Multi-Element Radio Linked Interferometer Network (MERLIN). The observations were performed on 7 May 2007, 4 June 2007 and 6 June 2007 for $\mathrm{OH} 37.1-0.7, \mathrm{OH} 12.8-0.9$ and W43A respectively. We achieved a spatial resolution of of $0.3 \times 0.2$ arcsec. The observations of W43A were presented earlier (A10). Here we present the observations of the two other sources, following the same procedure as we used for W43A.

For $\mathrm{OH} 37.1-0.8$, the observations were performed in full polarization spectral line mode with 256 spectral channels and a bandwidth of $0.25 \mathrm{MHz}$, covering a velocity width of $44 \mathrm{~km} \mathrm{~s}^{-1}$ and a channel width of $0.2 \mathrm{~km} \mathrm{~s}^{-1}$. The observations were interleaved with observations of the phase reference source, $1904+013$, in wide-band mode in order to obtain an optimal signal-to-noise-ratio. 3C 286 was observed as primary flux calibrator and polarization angle calibrator. 3C 84 was observed both in narrow band and wide band modes in order to apply band pass calibration and a phase offset correction. The rms noise in the emission free channels was $6.5 \mathrm{mJy}$.

Similarly, the observations of $\mathrm{OH}$ 12.8-0.9 were performed in full polarization spectral line mode. We also used a bandwidth of $0.25 \mathrm{MHz}$, but now with 512 spectral channels. This covers a velocity range of $\sim 44 \mathrm{~km} \mathrm{~s}^{-1}$ with a spectral resolution of $0.08 \mathrm{~km} \mathrm{~s}^{-1}$. We observed the phase calibrator 1829-207 in wide band mode, in order to obtain sufficient signal to noise ration. 3C 286 and 3C 84 were observed as a primary flux calibrator and polarization angle calibrator. The rms noise in emission free channels was $9.4 \mathrm{mJy}$.

The initial processing of the raw data and conversion to FITS file were performed using the local "d-programs" at Jodrell Bank. 3C 286 was used to obtain the flux density of the amplitude calibrator 3C 84. We used the Astronomical Image Processing Software Package (AIPS) to perform the rest of the calibration, editing and imaging of the data. We corrected the phase offset between the wide and narrow band data by determining the difference in phase solution for 3C 84. These solutions were then applied to phase reference source. The phase and amplitude corrections derived from the phase calibrator where applied to the target data set.

The polarization calibration for leakage was determined using 3C 84, and the $R$ and $L$ phase offset corrections were performed on $3 \mathrm{C} 286$. Image cubes were created for stokes $I, Q, U, V, R R$ and $L L$. A linearly polarized data cube was made using the Stokes $Q$ and $U$. Using the AIPS task SAD, $\mathrm{OH}$ maser features with peaks higher than three times the rms noise in the emission free channel were identified and fitted with a Gaussian in the total intensity image cube.

We use the cross-correlation method introduced by Modjaz et al. (2005) to measure the magnetic field due to the Zeeman splitting. In this method the right circular polarization (RCP) and the left circular polarization (LCP) spectra are cross-correlated to determine the velocity splitting. The magnetic field is determined by applying the Zeeman splitting coefficient to the measured velocity splitting. We refer to A10 for a detailed description of the polarization procedure.

\section{Results}

\section{1. $\mathrm{OH}$ maser observations of $\mathrm{OH} 12.8-0.9$}

Figure 1 shows the total intensity and the stokes $V$ spectra of $\mathrm{OH}$ 12.8-0.8. The stokes $I$ spectrum shows a double peak structure with peaks at -68.0 and $-43.7 \mathrm{~km} \mathrm{~s}^{-1}$, which is typical for the $\mathrm{OH}$ masers of AGB stars. Since maser lines are very narrow, it is likely that each of the peaks in the spectrum is a blend of several maser lines.

The properties of the $\mathrm{OH}$ maser features identified using the AIPS task SAD are shown in Table 1. Each maser feature consists of several maser spots. On average, we measured a magnetic field of $\sim-31 \mu \mathrm{G}$ and $\sim 27 \mu \mathrm{G}$ for the blue-shifted and red-shifted regions, respectively. The magnetic field measured for the blue- and red-shifted features have opposite signs which may indicate a clear difference between the blue- and redshifted regions. We found no significant linear polarization for the $\mathrm{OH}$ maser features of this source. Additionally, no stokes $V$ signal is apparent in the spectrum shown in Fig. 1, which corresponds to the total $V$ emission from all maser features. However, an individual analysis of the features identified with SAD using the cross-correlation method still produces 3-4 $\sigma$ detections of the magnetic field strength. This is confirmed by the $V$-spectra, shown in Fig. 2, taken at the location of one of the individual maser feature (feature 13). Now a circular polarization signal is clearly visible.

The spatial distribution of the blue- and red-shifted $\mathrm{OH}$ maser spots of $\mathrm{OH} 12.8-0.9$ are plotted in Fig. 1. The spots are 

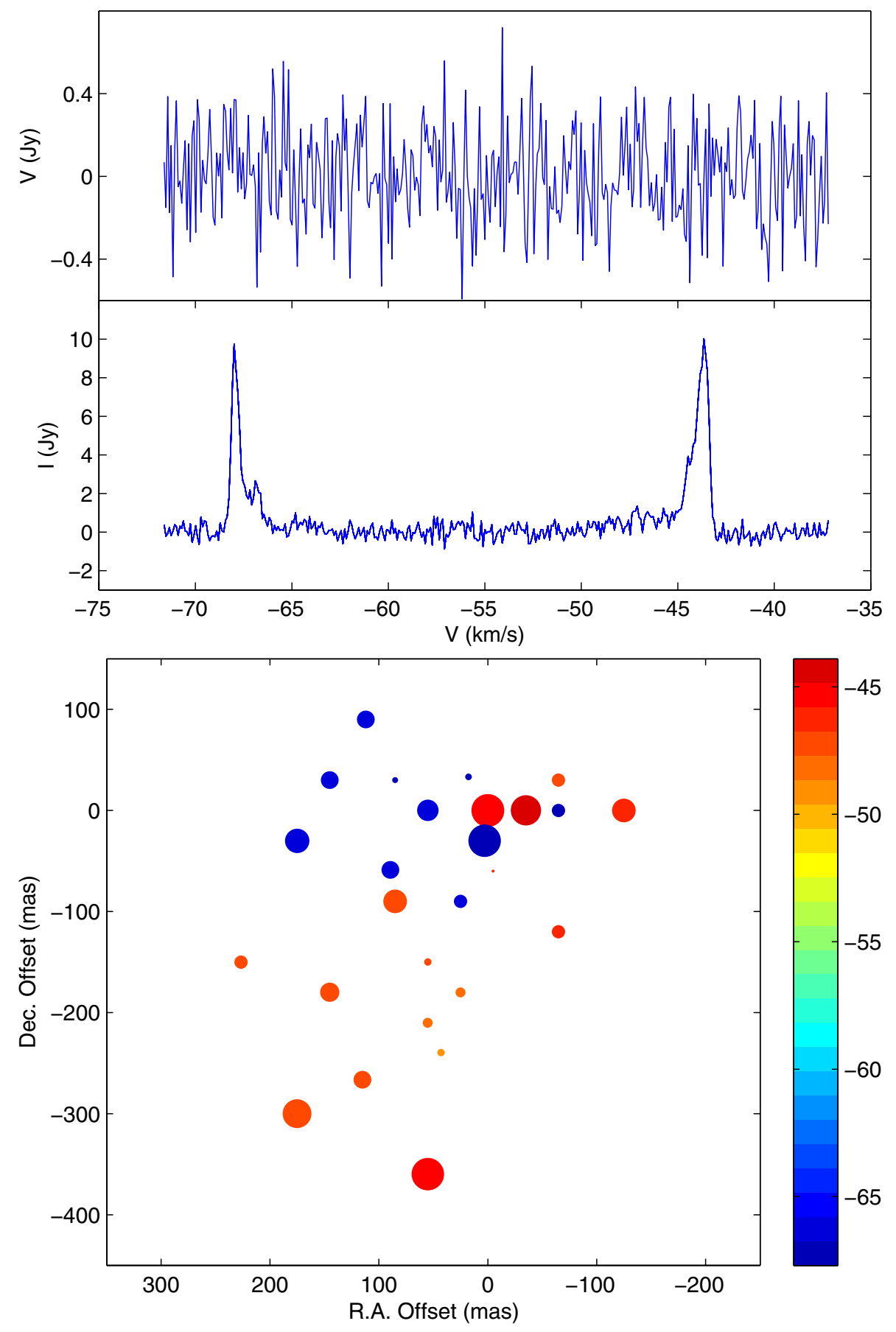

Fig. 1. Top panel: total power (I) and stokes $V$ spectra of the $\mathrm{OH}$ maser region of $\mathrm{OH} 12.8-0.9$. The $V$ spectrum is shown after removing the residual scaled down of the stokes $I$. Bottom panel: the $\mathrm{OH}$ maser region of $\mathrm{OH}$ 12.8-0.9. The offset positions are with respect to the maser reference feature 1 . The maser spots are color-coded according to their radial velocity. The size of the spots is scaled logarithmically according to their flux density.

color-coded according to their radial velocity. We calculated the average position for the red- and blue-shifted spots. The distribution of the spots implies that the maximum extent of the $\mathrm{OH}$ maser region and the offset between the blue- and red-shifted emission are $\sim 500$ mas and $\sim 135$ mas in the plane of the sky, respectively. The measured offset is slightly higher than the standard deviation in the spread of the red- and blue-shifted maser features $(\sim 100$ mas), and significantly larger than the positional uncertainty on the individual features ( $<30$ mas). The distance to $\mathrm{OH} 12.8-0.9$ is not accurately known, based on Galactic rotation models it was estimated to be $8 \mathrm{kpc}$ (Baud et al. 1985), but this could be off by a considerable amount. At a distance of $8 \mathrm{kpc}$, the measured maximum extent of the $\mathrm{OH}$ maser region of this source corresponds to 4000 AU. However, the actual extent of the $\mathrm{OH}$ maser region could be much larger, since we only detected emission at the blue- and red-shifted peaks and no emission was found close to the stellar velocity $\left(V_{\mathrm{lsr}}=-55 \mathrm{~km} \mathrm{~s}^{-1}\right)$. The observed distribution does not seem to be explained by the spherically expanding shell model, in which the red- and blueshifted peaks should be coincident.

\section{2. $\mathrm{OH}$ maser observations of $\mathrm{OH} 37.1-0.8$}

Figure 3 displays the total intensity and stokes $V$ spectra of $\mathrm{OH}$ 37.1-0.8. The spectrum shows a double peak structure. The emission is also detected in the circular polarization profile. The redshifted emission ranges from $73 \mathrm{~km} \mathrm{~s}^{-1}$ to $78 \mathrm{~km} \mathrm{~s}^{-1}$. The blueshifted emission ranges from $95 \mathrm{~km} \mathrm{~s}^{-1}$ to $103 \mathrm{~km} \mathrm{~s}^{-1}$. Each of the blue-shifted and red-shifted regions has multiple peaks and is a blend of several lines. In Table 2 we present the maser 
Table 1. Properties of the $\mathrm{OH}$ maser features of $\mathrm{OH} 12.8-0.9$.

\begin{tabular}{lccccc}
\hline \hline Feature & $\begin{array}{c}\text { RA } \\
1816\end{array}$ & $\begin{array}{c}\text { Dec } \\
-1815\end{array}$ & $\begin{array}{c}\text { Velocity } \\
\mathrm{km} \mathrm{s}^{-1}\end{array}$ & $\begin{array}{c}\text { Flux } \\
\text { Jy beam }^{-1}\end{array}$ & $\begin{array}{c}B \\
\mu \mathrm{G}\end{array}$ \\
\hline 1 & 49.21560 & 1.53 & -43.65 & 9.3 & $<19$ \\
2 & 49.21315 & 1.53 & -43.4 & 7.8 & $<19$ \\
3 & 49.21947 & 1.89 & -43.9 & 0.3 & $<37$ \\
4 & 49.21560 & 1.56 & -44.5 & 1.9 & $<22$ \\
5 & 49.21111 & 1.53 & -45.3 & 0.4 & $27 \pm 7$ \\
6 & 49.21947 & 1.62 & -45.7 & 0.6 & $<44$ \\
7 & 49.22368 & 1.80 & -46.2 & 0.3 & $<23$ \\
8 & 49.21947 & 1.74 & -46.6 & 0.5 & $<127$ \\
9 & 49.21862 & 1.77 & -47.5 & 0.6 & $<165$ \\
10 & 49.22189 & 1.59 & -65.3 & 0.3 & $<29$ \\
12 & 49.22579 & 1.50 & -66.2 & 0.3 & $<39$ \\
13 & 49.22347 & 1.44 & -67.9 & 0.3 & $-45 \pm 12$ \\
14 & 49.22158 & 1.50 & -66.7 & 1.5 & $-27 \pm 8$ \\
15 & 49.21685 & 1.50 & -66.9 & 1.6 & $-21 \pm 6$ \\
16 & 49.21581 & 1.56 & -67.6 & 9.1 & $<17$ \\
17 & 49.21105 & 1.53 & -66.9 & 0.8 & $<15$ \\
\hline
\end{tabular}

Notes. Each maser feature consists of several maser spots. The upper limits are given in absolute values.

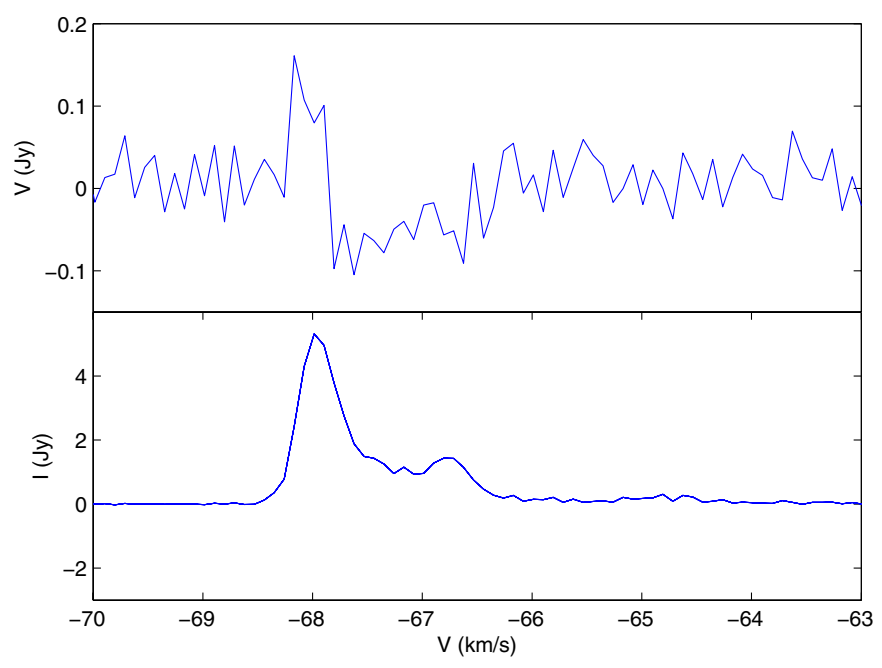

Fig. 2. Total intensity (bottom panel) and stokes $V$ (top panel) spectra of the blue shifted $\mathrm{OH}$ maser region of $\mathrm{OH}$ 12.8-0.9 taken at the position of feature 13. The $V$ spectrum is shown after removing the residual scaled down of the stokes $I$.

components that we identified for the $\mathrm{OH}$ maser region of this star, using the AIPS task SAD. Each component consists of several maser spots. We did not find significant linear polarization for the $\mathrm{OH}$ maser features of this source. On average we measured a magnetic field of $\sim 430 \mu \mathrm{G}$ and $\sim 230 \mu \mathrm{G}$ for blue- and red-shifted masers, respectively.

Figure 3 displays the spatial distribution of the $\mathrm{OH}$ maser spots of $\mathrm{OH}$ 37.1-0.8. We determined the average position for the red- and blue-shifted peaks. The maximum extent of the $\mathrm{OH}$ maser region and the offset between the blue- and red-shifted emission are $\sim 200$ mas and $\sim 100$ mas, respectively. The offset is almost twice higher than the standard deviation in the spread of the red- and blue-shifted maser features ( $\sim 50$ mas). The best estimate for the distance of this source based on galactic rotation model is $8 \mathrm{kpc}$ (Baud et al. 1985). At this distance, the measured maximum extent of the $\mathrm{OH}$ maser region of this source is $\sim 1600$ AU. This distribution is not consistent with spherically
Table 2. Properties of the $\mathrm{OH}$ maser features of $\mathrm{OH} 37.1-0.8$.

\begin{tabular}{lccccc}
\hline \hline Feature & $\begin{array}{c}\text { RA } \\
1902\end{array}$ & $\begin{array}{c}\text { Dec } \\
0320\end{array}$ & $\begin{array}{c}V \\
\mathrm{~km} \mathrm{~s}^{-1}\end{array}$ & $\begin{array}{c}\text { Peak flux } \\
\text { Jy }\end{array}$ & $\begin{array}{c}B \\
\mu \mathrm{G}\end{array}$ \\
\hline 1 & 6.28344 & 15.7047 & 102.3 & 1.9 & $180 \pm 3$ \\
2 & 6.28736 & 15.6984 & 102.6 & 5.0 & $180 \pm 2$ \\
3 & 6.29012 & 15.6579 & 101.6 & 3.1 & $240 \pm 3$ \\
4 & 6.2978 & 15.6186 & 101.4 & 2.1 & $260 \pm 5$ \\
5 & 6.29083 & 15.658 & 99.8 & 2.8 & $240 \pm 3$ \\
6 & 6.29074 & 15.6257 & 99.6 & 3.8 & $290 \pm 7$ \\
7 & 6.2800 & 15.66 & 77.3 & 4.2 & $150 \pm 4$ \\
8 & 6.28534 & 15.66 & 74.2 & 4.7 & $850 \pm 5$ \\
9 & 6.28801 & 15.66 & 74.02 & 2.5 & $260 \pm 7$ \\
10 & 6.28801 & 15.7 & 73.8 & 1.3 & $720 \pm 6$ \\
\hline
\end{tabular}

Notes. Each maser features consists of several maser spots.

symmetric shell model, since the blue-shifted and red-shifted maser features are not coincident in the plane of the sky.

\section{Analysis}

\subsection{Description of the kinematical reconstruction procedure}

The observations of the $\mathrm{H}_{2} \mathrm{O}$ masers in a number of waterfountain sources revealed that these masers are located in bipolar structures (e.g. Vlemmings et al. 2006; Boboltz \& Marvel 2005). Thus the spherically symmetric CSE is modified, for example by carving out polar cavities. In order to understand the geometry of the $\mathrm{OH}$ maser region of these sources, we performed a geometrical reconstruction of the masers in three dimensional grids. We did not consider radiative transfer of masers in our analysis. Instead, the velocity coherent path length along the line of sight was determined for maser shells of various geometries and velocity fields. In all cases, we assumed a maximum shell radius of $3 \times 10^{16} \mathrm{~cm}(\sim 2000 \mathrm{AU})$, which is typical for $\mathrm{OH}$ masers (Chapman \& Cohen 1986).

We assumed that the masers are saturated and that the resulting intensity increases linearly rather than exponentially with path-length. Observations indicate that the $1612 \mathrm{MHz}$ respond smoothly and linearly to the change in physical conditions and pump rates, which could indicate that the masers are saturated (Elitzur 1990). Therefore, the saturation assumption enables us to investigate the extent at which the velocity field along the line of sight contributes to the profile and structure of the maser shells, but not the line strengths in detail.

A similar analysis was performed by Bowers (1991), in which they obtained the velocity coherent path length along the line of sight for ellipsoidal shells with various inclinations from the plane of the sky. They also considered different velocity fields, incorporating isotropic velocity, rotation and radial acceleration. We use a similar modeling procedure, but we expand our work to more complex morphologies and velocity fields that may explain the distribution of $\mathrm{OH}$ masers in water-fountain sources which show significant deviation from spherically symmetric CSEs. For the basic method we refer to Bowers (1991).

We consider two different possibilities for the distribution of $\mathrm{OH}$ masers in water-fountain sources: equatorial distribution (Fig. 4) or biconical outflow (Fig. 5). In the case of equatorial distribution, the $\mathrm{OH}$ masers stem from the equatorial region of the circumstellar shell, while the $\mathrm{H}_{2} \mathrm{O}$ masers are located in a bipolar jet-like outflow. Alternatively, the $\mathrm{OH}$ masers may originate in a biconical outflow surrounding the $\mathrm{H}_{2} \mathrm{O}$ maser jet (Likkel et al. 1992). The spectral profile and emission structure 
$\mathrm{N}$. Amiri et al.: The kinematics and magnetic fields in water-fountain sources based on $\mathrm{OH}$ maser observations
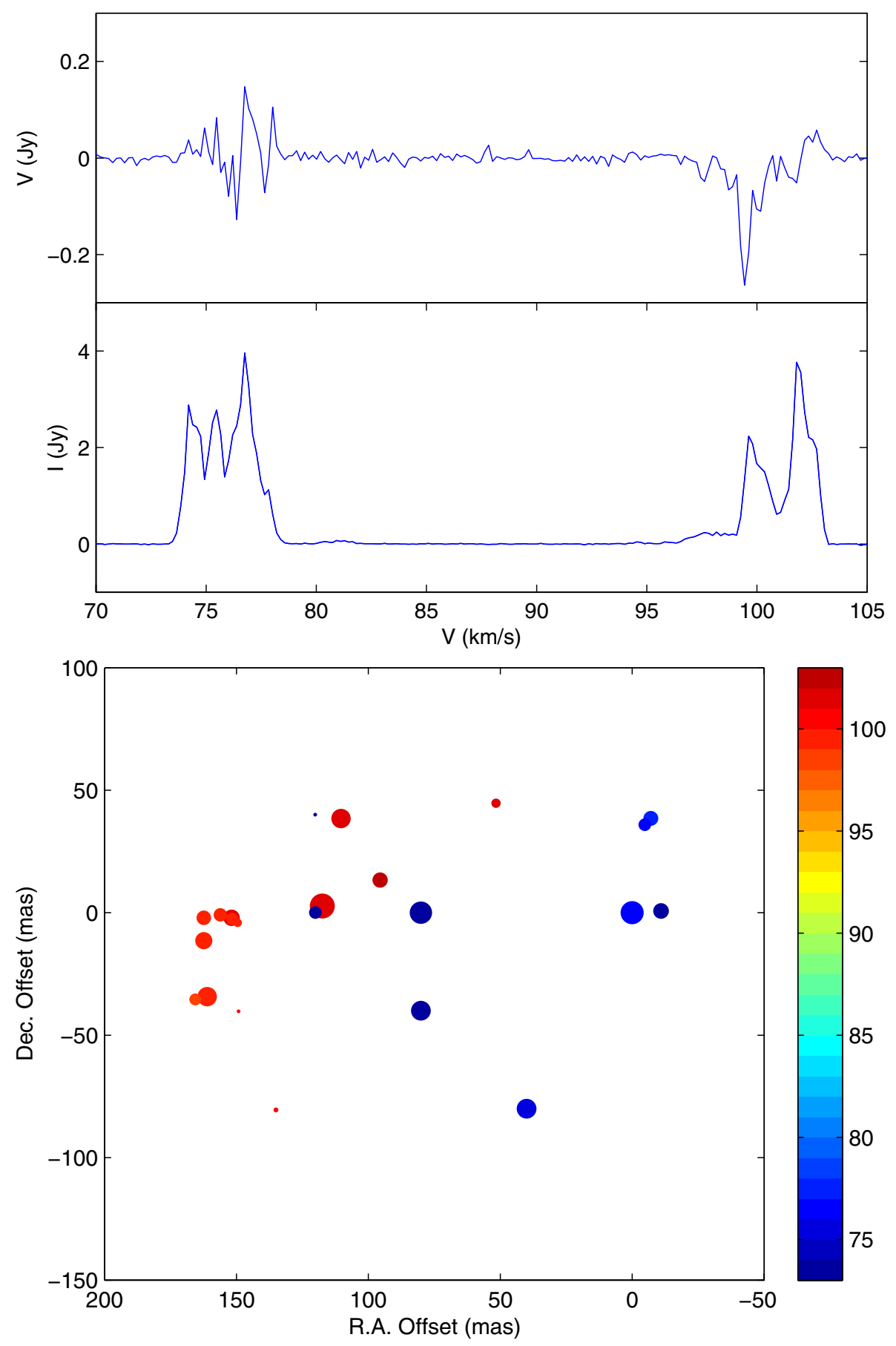

Fig. 3. Top panel: total power and the stokes $V$ spectra of the $\mathrm{OH}$ maser region of $\mathrm{OH} 37.1-0.8$. The $V$ spectrum is shown after removing the residual scaled down of the stokes I. Bottom panel: the $\mathrm{OH}$ maser region of $\mathrm{OH} 37.1-$ 0.8 . The offset positions are with respect to the maser reference feature 7 . The maser spots are color-coded according to their radial velocity. The size of the spots is scaled logarithmically according to their flux density.

were calculated assuming these two morphologies. Throughout our analysis the opening angle in the case of equatorial distribution is defined as the angle from the equatorial plane. In contrast, for a biconical outflow the opening angle is defined from the polar axis.

We incorporate two different velocity fields: isotropic expansion or azimuthally dependent velocity, either towards the equatorial plane or the polar axis. In the case of isotropic outflow, we assume a constant expansion velocity of $15 \mathrm{~km} \mathrm{~s}^{-1}$, which is typical for the $\mathrm{OH}$ maser in evolved stars (e.g. Sevenster 2002). Since the velocity scales linearly with the extent of the velocity, choosing other values of the expansion velocity does not change the profile shape in our models. In the case where the velocity is enhanced towards the equatorial plane, we use $V_{\exp }=a+b \times|\sin (\phi)|$. Similarly, if the velocity increases towards the polar axis, we adopt $V_{\exp }=a+b \times|\cos (\phi)|$. In both cases $a+b=15 \mathrm{~km} \mathrm{~s}^{-1}$. In all cases $\phi$ is the angle from the polar axis.

If the velocity has a latitudinal dependence, we assume an elongated ellipsoid geometry for the maser shell. We define the ellipticity $\left.\left(e=\sqrt{(} 1-\frac{R_{\min }^{2}}{R_{\max }^{2}}\right)\right)$ of the oblate or prolate shells by the velocity ratio between the major and minor axes of the ellipsoid

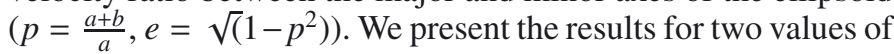
ellipticity ( $e=0.7$ and $e=0.9$ ), which implies that the velocity of the major axis of the ellipse is twice and four times higher than that for the minor axis of the ellipse. The models in this section have not been adjusted to match the data for a specific object.

The following plots are presented for each model:

1. normalized intensity profile versus velocity, which indicates the path length along the line of sight for each velocity 


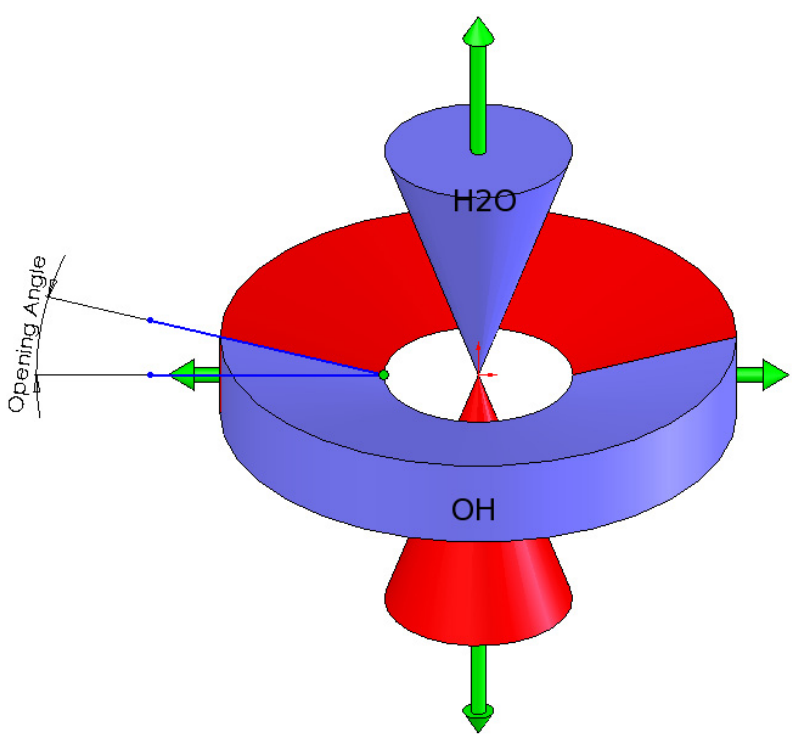

Fig. 4. Equatorial distribution of the $\mathrm{OH}$ masers surrounding the $\mathrm{H}_{2} \mathrm{O}$ maser jet in the CSEs of water-fountain sources. The opening angle is shown from the equatorial plane.

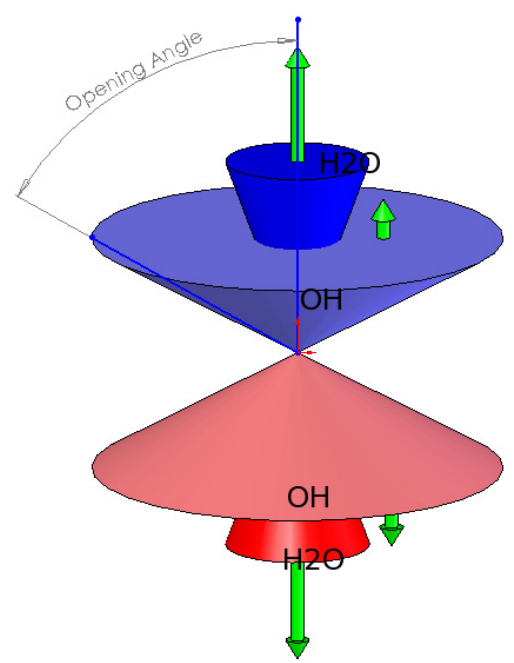

Fig. 5. Biconical distribution of the $\mathrm{OH}$ masers around the $\mathrm{H}_{2} \mathrm{O}$ maser jet in the CSEs of water-fountain sources. The opening angle is shown from the polar axis.

integrated over the sky. We calculated the spectra for inclinations of $0^{\circ}, 15^{\circ}, 30^{\circ}, 45^{\circ}, 60^{\circ}, 75^{\circ}, 90^{\circ}$ from the plane of the sky. At each inclination, the spectra are presented for $25^{\circ}$, $45^{\circ}, 65^{\circ}, 80^{\circ}$ opening angles;

2. the offset between the blue-shifted and red-shifted peaks in the plane of the sky. The spatial distribution of the $\mathrm{OH}$ maser region of a number of water-fountain sources reveal that the blue-shifted and red-shifted features are separated in the plane of the sky. For the cases where the spectrum shows the typical double peak profile, we calculated the position of the blue-shifted and red-shifted peaks and measured the offset. For more complex spectra where the spectra show inner peaks it becomes more complicated as the position of the inner peaks and outer peaks are different. Therefore, we measured the position of the outer peaks for the blue-shifted and red-shifted emission and determined the offset value. For example, Fig. 6 shows the distribution of the blue-shifted and red-shifted peaks in the case where masers are confined

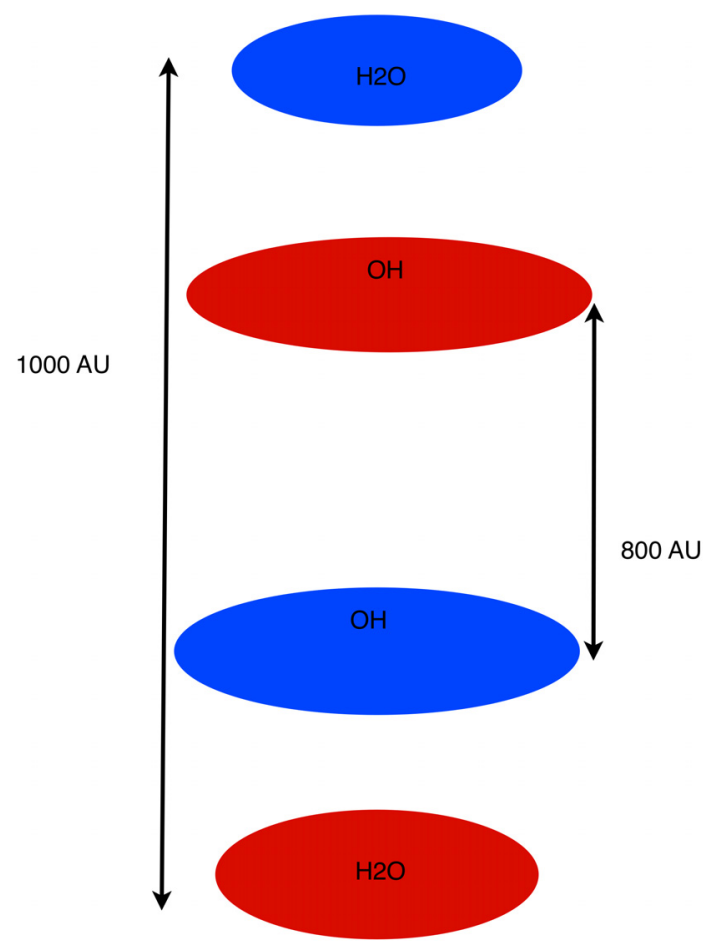

Fig. 6. The offset between the blue-shifted and red-shifted peaks of maser emission calculated from the models. The $\mathrm{OH}$ masers are confined within $30^{\circ}$ from the equatorial plane with an inclination of $40^{\circ}$ from the plane of the sky. The models show that the $\mathrm{OH}$ masers are reversed in the plane of the sky compared to those of the bipolar $\mathrm{H}_{2} \mathrm{O}$ maser outflow.

within $30^{\circ}$ from the equatorial plane with an inclination of $40^{\circ}$ from the sky plane, which shows that the $\mathrm{OH}$ masers are reversed in the plane of the sky compared to those of the bipolar outflow. Similarly, for all cases we determined the separation between the blue shifted and red shifted regions. The negative sign for the offset values implies that the masers are located in the opposite direction with respect to the fast bipolar $\mathrm{H}_{2} \mathrm{O}$ maser outflow.

We consider different scenarios, which are intended as a guide for analysis of the $\mathrm{OH}$ masers in water-fountain sources. The plots presented are particularly useful for comparison with the observed parameters of spectral structure and spatial distribution in these objects. Figures 7 and 8 show the results for the cases of equatorial and biconical distribution of the masers, where the expansion velocity is constant in all directions. For the other cases where the velocity has latitudinal dependence we present the result of our modeling calculations in Figs. 6-19.

\section{A1: Equatorial distribution, $V_{\exp }=$ const.}

This case describes the situation that the $\mathrm{OH}$ masers remain active in the equatorial plane, while $\mathrm{H}_{2} \mathrm{O}$ masers originate in a high velocity outflow in the polar region. The outflow velocity is constant in all directions. The $\mathrm{OH}$ masers in this scenario would have a slower expansion velocity with respect to the fast outflow in the polar region.

Figure 7 displays the results for this case. The spectra show double peak structure for inclinations below $45^{\circ}$ from the plane of the sky. For $60^{\circ}$ and $75^{\circ}$ inclinations, the spectra display inner peaks for opening angles smaller than $45^{\circ}$. At $90^{\circ}$, the spectra again show double peaks. 
N. Amiri et al.: The kinematics and magnetic fields in water-fountain sources based on $\mathrm{OH}$ maser observations
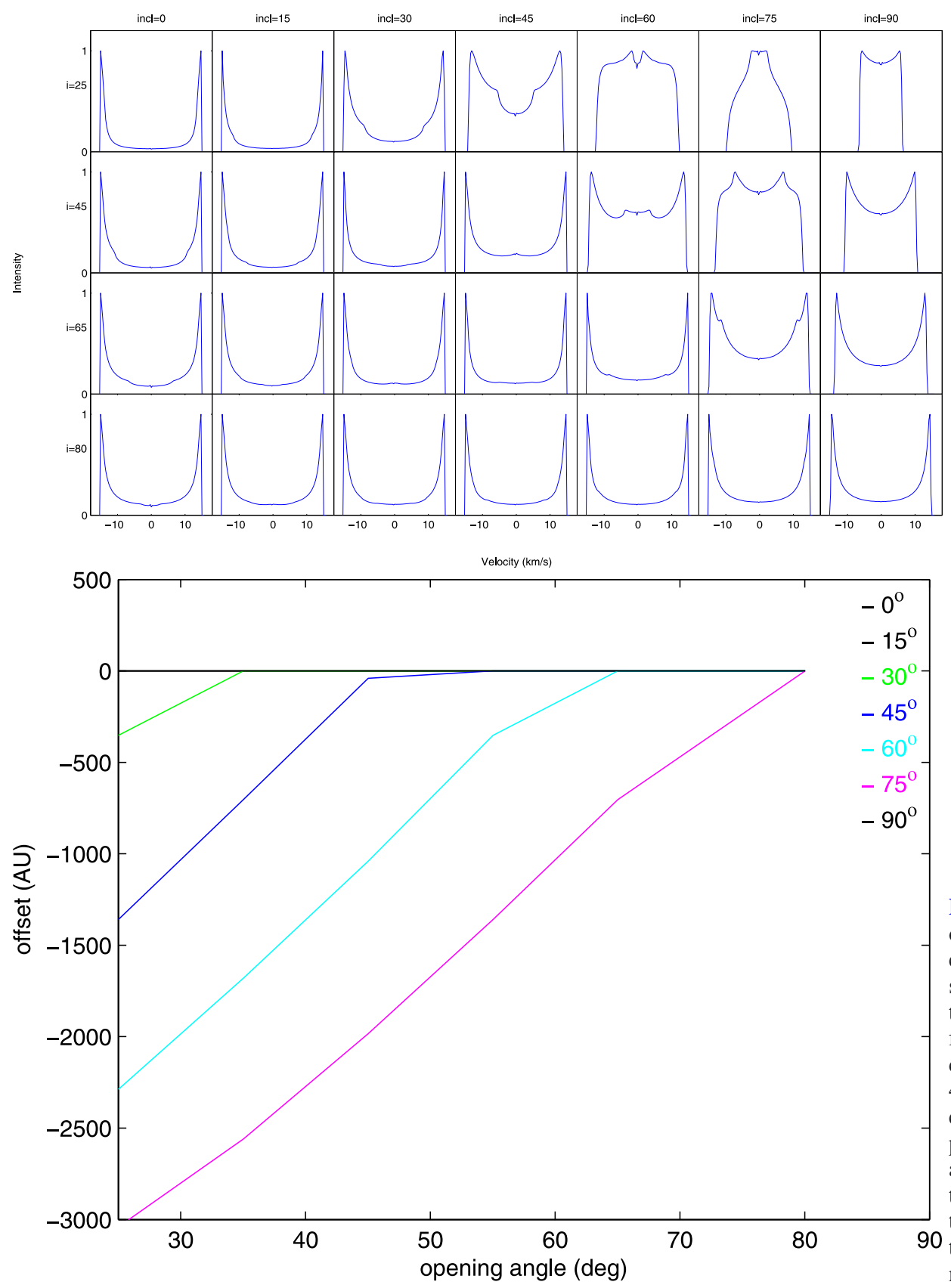

Fig. 7. Model calculations for the case of isotropic outflow of $15 \mathrm{~km} \mathrm{~s}^{-1}$ with equatorial distribution. The top panel shows the spectra calculated for inclinations of $0^{\circ}, 15^{\circ}, 30^{\circ}, 45^{\circ}, 60^{\circ}, 75^{\circ}, 90^{\circ}$ from the plane of the sky. For each inclination the spectra are shown for $25^{\circ}$, $45^{\circ}, 65^{\circ}$ and $80^{\circ}$ opening angle from the equatorial plane. The bottom panel displays the offset between the blue shifted and red shifted regions in the plane of the sky. The negative sign indicates that the masers are reversed in the plane of

90 the sky compared to those located in a narrow region in the polar axis.

The diagram at the bottom shows the offset between the blue-shifted and red-shifted regions versus opening angle from the equatorial plane. Each line shows the offset at a specific inclination. For $0^{\circ}, 15^{\circ}$ and $90^{\circ}$ inclination angle from the plane of the sky, the separation is zero and the blue-shifted and red-shifted emission coincide in the plane of the sky. For other inclinations the masers are reversed in the sky plane. The offset is highest at small opening angles and as expected approaches 0 for large opening angles in the equatorial region. The highest offset is $\sim 3000$ AU.

\section{A2: Equatorial distribution, $V_{\exp }=a+b \times|\sin (\phi)|$}

Similar to case A1, we assume equatorial distribution for the masers. But we adopt a latitudinal dependent velocity of $V_{\exp }=$ $a+b \times|\sin (\phi)|$, where the velocity is highest in the equatorial plane and decreases towards the polar region. The shell geometry is elongated towards the equatorial plane. This case potentially describes the situation where the masers are affected by a binary companion in the equatorial region of the CSE.

We calculated the spectra and map structure for $e=0.7$ and $e=0.9$. The results of this case are shown in Figs. 9 and 10 . Comparison of the spectra obtained for this scenario and those for the case A1, shows that the shape of the spectra remains similar at inclinations below $60^{\circ}$, where the spectra show double peaks. Above $60^{\circ}$ inclination the inner peaks occur, which dominate the emission at the edges of the spectrum for the case of $e=0.9$.

Again, the second figure displays the offset value between the blue-shifted and red-shifted peaks. At $0^{\circ}$ and $90^{\circ}$ inclinations from the plane of the sky, the separation is zero. For other inclinations the masers are reversed and the offset is in the range 400-2500 AU and 400-2500 AU for the cases of $e=0.7$ and $e=0.9$, respectively. 

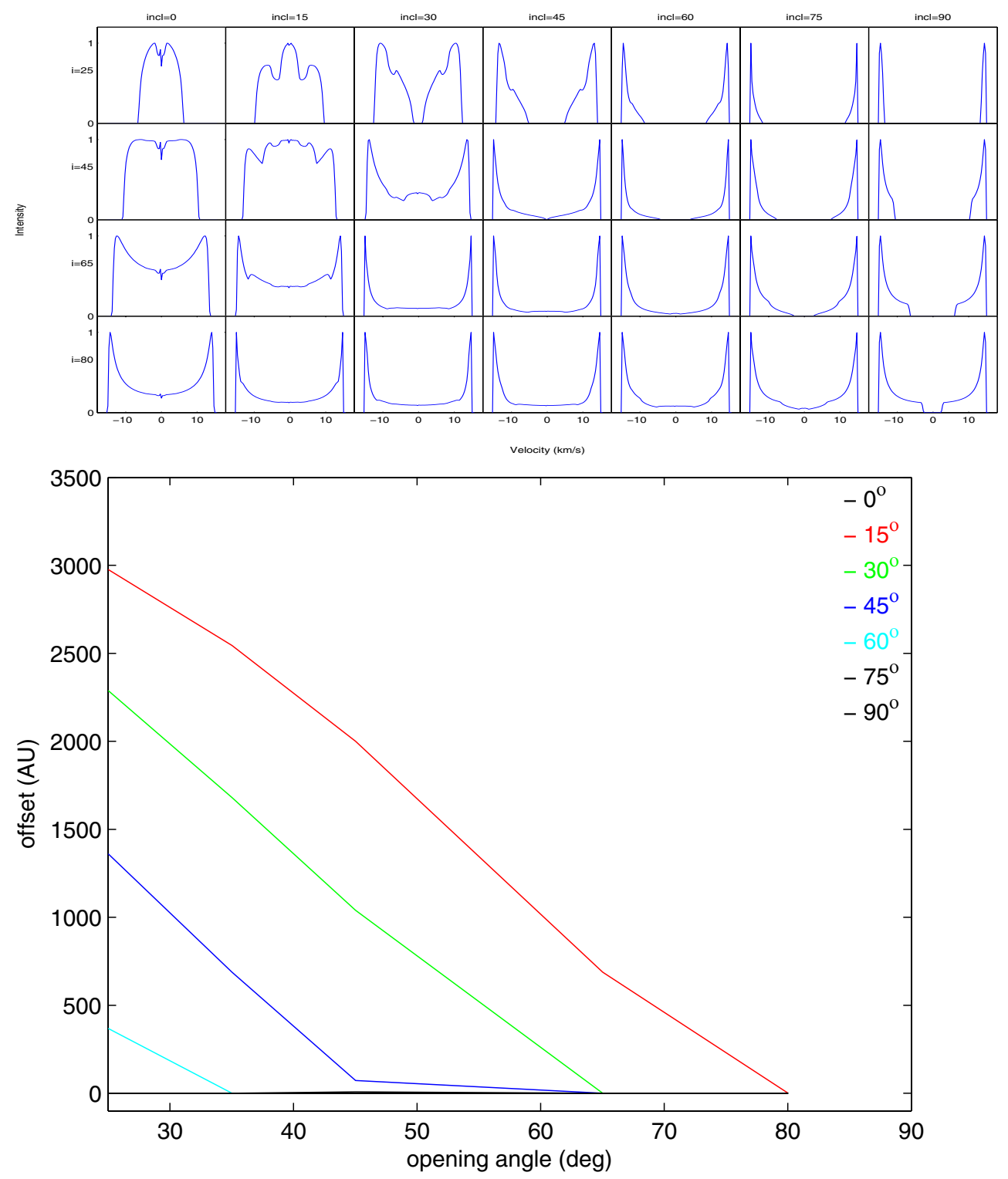

Fig. 8. Model calculations for the case of biconical outflow with constant expansion velocity of $15 \mathrm{~km} \mathrm{~s}^{-1}$.

\section{A3: Equatorial distribution, $V_{\exp }=a+b \times|\cos (\phi)|$}

In this case we assumed equatorial distribution for the masers but the shell geometry is considered to be elongated towards the polar axis. The velocity field is latitudinally dependent $\left(V_{\exp }=\right.$ $a+b \times|\cos (\phi)|)$, which implies that the velocity increases towards the polar region.

We calculated the models for $e=0.7$ and $e=0.9$, the results of which are shown in Figs. 11 and 12. The spectra show significant differences compared to the case A1, where the velocity is constant in all directions. For all inclinations the spectra show inner peaks, which are even higher than the emission at the edges of the spectrum for opening angles smaller than $45^{\circ}$.

The bottom figure shows the separation between the blue shifted and red shifted regions. For $0^{\circ}$ and $90^{\circ}$ inclination from the plane of the sky, the offset between the blue shifted and red shifted regions is zero. At $45^{\circ}, 60^{\circ}$ and $75^{\circ}$ inclinations the masers are reversed when the opening angle is smaller than $45^{\circ}, 60^{\circ}$ and $75^{\circ}$ from the equatorial plane, respectively. The maximum offset value is $\sim 1500$ AU and $\sim 800$ AU for the cases of $e=0.7$ and $e=0.9$, respectively.
Biconical distribution, $V_{\exp }=$ const.

A biconical distribution is another possibility for the distribution of $\mathrm{OH}$ masers in water-fountain sources (Likkel et al. 1992; Sahai et al. 1999). In this case, the OH masers are located in a biconical outflow surrounding the $\mathrm{H}_{2} \mathrm{O}$ maser jet, but at lower latitudes (Fig. 5) with a constant expansion velocity of $15 \mathrm{~km} \mathrm{~s}^{-1}$.

Figure 8 displays the results for this case. The spectra show double peak structures generally. However, at inclinations of $0^{\circ}$, $15^{\circ}$ and $30^{\circ}$ there are strong inner peaks for small opening angles $(i \leq 45)$.

For $0^{\circ}$ and $90^{\circ}$ inclination angle from the sky plane the offset between the blue-shifted and red-shifted peaks is zero. In contrast to case A1, the masers are not reversed in the plane of the sky. The maximum offset value is $3000 \mathrm{AU}$.

\section{B2: biconical distribution, $V_{\exp }=a+b \times|\sin (\phi)|$}

In this scenario, the masers are distributed in a biconical outflow and the expansion velocity increases azimuthally towards the equatorial region. This case describes the situation where the 
masers are affected by a binary companion in the equatorial region, while they are still entrained by the fast collimated outflow in the polar region.

We calculated the models for $e=0.7$ and $e=0.9$. The results of this case are shown in Figs. 13 and 14. Comparison of the spectra obtained for this case and those for the case B1 show significant differences in the profile structure for inclinations above $45^{\circ}$, where most of the spectra show inner peak emission.

The bottom figure shows the offset between the blue-shifted and red-shifted emission. The masers are reversed for large inclinations from the plane of the sky, in particular at $45^{\circ}, 60^{\circ}$ and $75^{\circ}$. The maximum offset value is $\sim 1500 \mathrm{AU}$ and $\sim 800 \mathrm{AU}$ for $e=0.7$ and $e=0.9$ respectively.

\section{B3: biconical distribution, $V_{\exp }=a+b \times|\cos (\phi)|$}

In this case, the masers are distributed in a biconical outflow and the velocity has latitudinal dependence towards the polar axis. Figures 15 and 16 show the results for $e=0.9 e=0.9$ cases.

For $45^{\circ}$ to $90^{\circ}$ inclination angle from the plane of the sky, the spectra show double peak profiles, however for $0^{\circ}$ to $30^{\circ}$ inclinations the spectra show inner peaks. The offset between the blue shifted and red shifted regions is higher for smaller opening angles and approaches zero for higher opening angles. The maximum offset value is $\sim 2500 \mathrm{AU}$ and $\sim 1600 \mathrm{AU}$ for $e=0.7$ and $e=0.9$ respectively.

\subsection{Application to individual sources}

\subsubsection{W43A}

W43A is a unique water-fountain source because it exhibits $\mathrm{OH}$ (1612, 1665 and $1667 \mathrm{MHz}$ ), $\mathrm{H}_{2} \mathrm{O}$ and $\mathrm{SiO}$ masers (A10; Nyman et al. 1998; Imai et al. 2002). The spectra of the $\mathrm{OH}$ and $\mathrm{H}_{2} \mathrm{O}$ masers of this object exhibit double peak profiles. The $\mathrm{H}_{2} \mathrm{O}$ maser velocity range $\left(\sim 180 \mathrm{~km} \mathrm{~s}^{-1}\right)$ is much higher than that for the $\mathrm{OH}$ masers $\left(\sim 13 \mathrm{~km} \mathrm{~s}^{-1}\right)$, which makes this the archetype water-fountain source (Imai et al. 2002; Likkel et al. 1992).

High resolution interferometric observations of the $\mathrm{H}_{2} \mathrm{O}$ maser region of $\mathrm{W} 43 \mathrm{~A}$ revealed a highly collimated and precessing jet. The $\mathrm{H}_{2} \mathrm{O}$ masers are found in two opposing clusters separated by $\sim 1700 \mathrm{AU}$ at a distance of $2.6 \mathrm{kpc}$ (Diamond et al. 1985). The $\mathrm{H}_{2} \mathrm{O}$ maser jet has an outflow velocity of $\sim 145 \mathrm{~km} \mathrm{~s}^{-1}$ with $39^{\circ}$ inclination from the plane of the sky (Imai et al. 2002). The $\mathrm{SiO}$ masers of this source are thought to occur in a shock at the interface between the fast collimating jet and the slow biconical outflow (Imai et al. 2005). The spatial distribution of the $\mathrm{OH}$ maser region of W43A revealed that the $\mathrm{OH}$ masers are located inside the $\mathrm{H}_{2} \mathrm{O}$ maser region. The $\mathrm{OH}$ masers are found in two opposing clusters separated by $\sim 650 \mathrm{AU}$. The red-shifted and blue-shifted $\mathrm{OH}$ masers are reversed in the plane of the sky with respect to those of $\mathrm{H}_{2} \mathrm{O}$ masers (A10).

The fact that the blue and red-shifted $\mathrm{OH}$ maser features are not positionally coincident in the plane of the sky, is not consistent with an isotropically expanding shell model. Additionally, the bipolar structure of the $\mathrm{H}_{2} \mathrm{O}$ and $\mathrm{SiO}$ maser regions is another piece evidence that these masers are located in aspherical shells.

The expansion velocity of the $\mathrm{OH}$ maser region of $\mathrm{W} 43 \mathrm{~A}$ is $\sim 18 \mathrm{~km} \mathrm{~s}^{-1}$ (A10). This seems to imply that the $\mathrm{OH}$ maser region is not affected by the fast post-AGB wind. We considered the two scenarios of either an equatorial distribution or a biconical outflow, as explained in Sect. 4.1 and compared the spectral profile and the $\mathrm{OH}$ masers spatial distribution with the model calculations. The same inclination $\left(39^{\circ}\right)$ as that of the collimated $\mathrm{H}_{2} \mathrm{O}$ maser jet was adopted for the $\mathrm{OH}$ maser region (Imai et al. 2002).

If the masers are distributed in the equatorial plane of the circumstellar shell with a constant expansion velocity in all directions, then our calculations show that the masers are reversed in the plane of the sky and the spectra would show double peak structures for all opening angles in the equatorial region (Fig. 7). For an opening angle of $\sim 35^{\circ}$, the offset corresponds to the observed value of $\sim 650 \mathrm{AU}$. If the velocity is azimuthally enhanced towards the equatorial plane, the spectra would show double peak profiles in all opening angles (Figs. 9 and 10). The models show that the masers are reversed in the plane of the sky, and the offset is in the range $400 \mathrm{AU}$ to $700 \mathrm{AU}$ for all opening angles in the equatorial plane. Therefore, this case may also describe the distribution of the $\mathrm{OH}$ masers of W43A.

On the other hand if the velocity has latitudinal dependence enhanced towards the polar axis, the spectra show inner peaks (Fig. 12). These profile shapes are not consistent with the $\mathrm{OH}$ maser profile of W43A. Therefore, this scenario is unlikely for the $\mathrm{OH}$ maser region of W43A.

Alternatively, we considered biconical distribution of the $\mathrm{OH}$ maser region of W43A. If the velocity is isotropic or azimuthally enhanced towards the polar axis, the masers are not reversed in the plane of the sky (Figs. 8 and 16), which is not consistent with the $\mathrm{OH}$ maser morphology of W43A. However, if the velocity is azimuthally enhanced towards the equatorial region, the spectra show double peak profiles at opening angles larger than $70^{\circ}$, for which the separation between the blue shifted and red shifted regions is $\sim 650 \mathrm{AU}$ (Fig. 14). Therefore, this scenario may also explain the $\mathrm{OH}$ maser morphology of W43A. However, for such large opening angles we are approaching an oblate distribution with enhanced outflow in the equatorial region. Thus, in either case, the reconstruction of the $\mathrm{OH}$ maser region of W43A suggests a predominantly equatorial distribution.

\subsection{2. $\mathrm{OH} 12.8-0.9$}

$\mathrm{OH} 12.8-0.9$ is a water-fountain candidate. This source was first reported by Baud et al. (1979) as a type II OH/IR star on the basis of its double peak $1612 \mathrm{MHz} \mathrm{OH}$ maser profile. The source IRAS 18316-1816 is identified as the IR counterpart to this source. The $\mathrm{H}_{2} \mathrm{O}$ masers of $\mathrm{OH} 12.8-0.9$ were first discovered by Engels et al. (1986). They also found that the $\mathrm{OH}$ masers of this object lie inside the $\mathrm{H}_{2} \mathrm{O}$ maser velocity range, which is not typical for $\mathrm{OH} / \mathrm{IR}$ stars. VLBI $\mathrm{H}_{2} \mathrm{O}$ maser observations of this object revealed that the masers are located in two opposing clusters at the tips of a bipolar jet-like structure oriented north-south (Boboltz \& Marvel 2005). The blue-shifted and red-shifted $\mathrm{H}_{2} \mathrm{O}$ masers are found in arc-shape structures separated by $\sim 110$ mas in the plane of the sky.

Multi-epoch VLBA observations revealed a 3D outflow velocity of $58 \mathrm{~km} \mathrm{~s}^{-1}$ for the $\mathrm{H}_{2} \mathrm{O}$ maser jet with $24^{\circ}$ inclination from the plane of the sky (Boboltz \& Marvel 2007). Comparison of the spatial morphology of the $\mathrm{OH}$ masers (Fig. 1) and the $\mathrm{H}_{2} \mathrm{O}$ masers reveal that the $\mathrm{OH}$ masers are located outside the $\mathrm{H}_{2} \mathrm{O}$ maser jet. The maximum extent of the $\mathrm{OH}$ maser region is $\sim 500$ mas, which is much larger than that for $\mathrm{H}_{2} \mathrm{O}$ masers ( $\sim 10$ mas). This is in contrast to Boboltz \& Marvel (2005) expectation that the $\mathrm{OH}$ masers should be located inside the $\mathrm{H}_{2} \mathrm{O}$ maser jet. Since this object is characterized as a high mass-loss source it is possible that this source is still in the AGB phase and 
that the jet has recently turned on in this source (Deacon et al. 2007).

We compared the observational parameters of the profile shape and spatial distribution (Fig. 1) with our model calculations. The $\mathrm{OH}$ maser spectrum of this source shows a double peak structure. Our observations show that the separation between the blue shifted and red shifted $\mathrm{OH}$ maser region of this source is $\sim 135$ mas (Fig. 1). Assuming a distance of $8 \mathrm{kpc}$ for this source, the offset corresponds to 1080 AU. Unlike W43A, the blue-shifted and red-shifted $\mathrm{OH}$ masers are not reversed in the plane of the sky compared to those of $\mathrm{H}_{2} \mathrm{O}$ masers.

The equatorial distribution of the masers is unlikely. Our models show that the masers are reversed in the plane of the sky at $24^{\circ}$ in the case of isotropic outflow and latitudinal dependent velocity enhanced towards the equatorial region (Figs. 7, 10). However, in the case where the velocity azimuthally increases towards the polar region, the masers are not reversed in the plane of the sky, but the spectral shapes in this case are quadruply peaked (Fig. 12), which is not consistent with the observed spectrum of this source.

If the masers are located in a biconical outflow with isotropic expansion velocity or azimuthal dependent velocity towards the polar axis, our results show that at opening angles of $\geq 65^{\circ}$, the spectra show double peak profiles (Figs. 8 and 16). However, the offset between the blue shifted and red shifted regions is smaller than 1000 AU. This implies that either the distance of $8 \mathrm{kpc}$ is not accurate for this source or the assumption of maximum shell radius of $2000 \mathrm{AU}$ is not correct. In the case of a biconical distribution where the velocity enhances towards the equatorial region (Fig. 14), at opening angles above $65^{\circ}$ the profile shape is double peaked, however the offset approaches zero at these large opening angles, which is not consistent with the observed spatial distribution of the $\mathrm{OH}$ maser region of this source. Therefore, a biconical outflow with isotropic expansion velocity or azimuthal dependent velocity towards the polar axis may explain the $\mathrm{OH}$ maser morphology of this source. However, we do not consider other effects (e.g. anisotropic pumping, etc.), that may produce the observed asymmetry of the $\mathrm{OH}$ maser region of this source.

\subsection{3. $\mathrm{OH} 37.1-0.8$}

The $\mathrm{H}_{2} \mathrm{O}$ masers of this source were first discovered by Engels et al. (1986) and the $\mathrm{OH}$ masers were first detected by Winnberg et al. (1975). The $\mathrm{H}_{2} \mathrm{O}$ maser velocity range of this source $\left(63-114 \mathrm{~km} \mathrm{~s}^{-1}\right.$; Engels 2002) is higher than that for $\mathrm{OH}$ masers $\left(73-103 \mathrm{~km} \mathrm{~s}^{-1}\right)$, which establishes the water-fountain nature of this source.

The spatial distribution of the $\mathrm{OH}$ maser of this source shows that these masers are located in aspherical shell. The separation between the two corresponds to $\sim 100$ mas (Fig. 3). Since no high resolution map of the $\mathrm{H}_{2} \mathrm{O}$ masers of this source is available, the spatial distribution of the $\mathrm{OH}$ maser features with respect to $\mathrm{H}_{2} \mathrm{O}$ masers is not clear in this source. Additionally, we do not have information on the orientation of the jet from the plane of the sky. Therefore, we can not easily distinguish between the equatorial or biconical outflow scenarios for the $\mathrm{OH}$ maser region of this source.

However, the $\mathrm{OH}$ maser spectrum of this source shows double peaks, but each peak is a blend of several maser lines. This could raise two possibilities. These peaks could originate from several maser spots with different velocities. Alternatively, geometric effects can produce this profile structure. Our calculations show that multiple peaks in the spectra may form in both biconical outflow and equatorial distribution; for example Figs. 12, 14.

\section{Discussions}

Our observations reveal that the $\mathrm{OH}$ maser region of the three water-fountain sources studied in this work show signs of nonspherical expansion. This morphology can not be explained by the standard spherically expanding shell model for the $\mathrm{OH}$ maser shells in evolved stars. The dynamical ages of the $\mathrm{H}_{2} \mathrm{O}$ maser jets of W43A \& OH 12.8-0.9 are 90 and 50 yr, respectively (Boboltz \& Marvel 2007; Imai et al. 2005). We measured a shell size of $>1600 \mathrm{AU}$ and the expansion velocity of $<15 \mathrm{~km} \mathrm{~s}^{-1}$ for the $\mathrm{OH}$ masers. This implies the dynamical time scale of $\sim 506 \mathrm{yr}$. This indicates that the $\mathrm{OH}$ maser region is significantly older compared to the $\mathrm{H}_{2} \mathrm{O}$ maser jet. Therefore, the collimating agent was likely already at work before the $\mathrm{H}_{2} \mathrm{O}$ maser jet was launched. $\mathrm{No} \mathrm{H}_{2} \mathrm{O}$ maser image of $\mathrm{OH} 37.1-0.8$ is available. Therefore we can not confirm whether the jet is older that the $\mathrm{OH}$ maser shell in this star. Alternatively, the observed asymmetry may not reflect an actual asymmetry in the distribution, but rather other effects such as anisotropic pumping.

Recent observations have shown that magnetic fields could have an important role in collimating the $\mathrm{H}_{2} \mathrm{O}$ maser jets in water-fountain sources. Vlemmings et al. (2006) show that the $\mathrm{H}_{2} \mathrm{O}$ maser jet of W43A is magnetically collimated. The bipolar $\mathrm{H}_{2} \mathrm{O}$ masers, can be explained by the dynamo-driven magnetic field. Blackman et al. (2001) showed that a single star model can produce magnetic driven dynamo in the AGB phase. However, since magnetic field drains rotational energy, it needs to be reseeded. A binary companion can maintain the differential rotation required during the lifetime of the AGB phase. In the presence of a binary companion, the in-spiral of the companion into the envelope produces rotational energy needed for the generation of the magnetic field (Nordhaus \& Blackman 2006).

Magnetic fields could also have an important role on the non-spherical shape of the $\mathrm{OH}$ masers in water-fountain sources. From our MERLIN observations, we measured a magnetic field of $\sim 430 \mu \mathrm{G}$ and $\sim 230 \mu \mathrm{G}$ for blue shifted and red shifted masers of OH 37.1-0.8, respectively. These values are approximately an order of magnitude larger than those measured for the $\mathrm{OH}$ maser features of $\mathrm{OH} 12.8-0.9(\sim 27 \mu \mathrm{G}$ and $\sim-31 \mu \mathrm{G}$ for the blueshifted and red-shifted regions, respectively). Previous MERLIN observations of the $\mathrm{OH}$ masers of W43A also revealed a field strength of $100 \mu \mathrm{G}$ (A10), which indicates that a large scale magnetic field is present in the circumstellar environment of this object. A10 discussed several non-Zeeman effects which could produce the observed circular polarization. These effects include observational and instrumental effects. However, they did not find any effects which could falsely be attributed to the Zeeman splitting. This implies that the circular polarization observed in the $\mathrm{OH}$ masers originates from the Zeeman splitting.

The fact that the field strength measured for $\mathrm{OH} 37.1-0.8$ is almost an order of magnitude larger than that for $\mathrm{OH} 12.8-0.9$ could imply that the $\mathrm{OH}$ maser region of $\mathrm{OH} 37.1-0.8$ is located at a smaller distance to the central star than that of $\mathrm{OH} 12.8$ 0.9. As shown in Figs. 1 and 3, the maximum extent of the $\mathrm{OH}$ maser region of OH37.1-0.8 is $\sim 200$ mas, which is $\sim 2.5$ times smaller than the $\mathrm{OH}$ maser region of $\mathrm{OH} 12.8-0.9$ ( $\sim 500$ mas). The magnetic field strength changes as a function of distance from the central star (Vlemmings et al. 2005, Fig. 6). The dipole field has $r^{-3}$ dependence and the solar type field has $r^{-2}$ dependence, where $r$ is the distance from the central star. The distance 
to these stars is poorly known. However, considering the maximum extent of the $\mathrm{OH}$ maser region of these objects, it could be due to the difference in size of the $\mathrm{OH}$ maser shell that we measure different field strengths. Therefore, depending on the magnetic field morphology, the field strength in the $\mathrm{OH}$ maser region of $\mathrm{OH} 37.1-0.8$ could be higher than that of $\mathrm{OH} 12.8-0.9$ by up to an order of magnitude.

We performed geometrical reconstruction in order to clarify the morphology of the $\mathrm{OH}$ maser region in waterfountain sources. We have studied the effects of many different parameters including the spectral shape, velocity field and the equatorial or biconical morphology. Our analyses show that the $\mathrm{OH}$ maser spots of $\mathrm{W} 43 \mathrm{~A}$ are likely located in the equatorial region of the circumstellar shell, while in $\mathrm{OH} 12.8-0.9$ the $\mathrm{OH}$ masers are likely found in a biconical outflow surrounding the $\mathrm{H}_{2} \mathrm{O}$ maser jet. The equatorial enhancement could be produced by the presence of a binary companion close to the CSE via common envelope (CE) evolution (Nordhaus \& Blackman 2006). Under certain conditions the Roche lobe overflow, in a binary system may result in both companions immersed in a CE (Paczynski 1976). Numerical simulations showed that a binary induced equatorial outflow is confined to opening angles of $20^{\circ}-$ $30^{\circ}$ (Terman \& Taam 1996).

Although the binary companion could explain the equatorial enhancement, at the same time it may destroy the maser action. Schwarz et al. (1995) performed statistical analysis for a limited sample of symbiotics, most of them containing Mira variables as primaries. Their analysis showed that for very wide systems $(R \geq 50 \mathrm{AU})$, all masers $\left(\mathrm{OH}, \mathrm{H}_{2} \mathrm{O}\right.$ and $\left.\mathrm{SiO}\right)$ can operate. At intermediate orbits (10 AU $\leq R \leq 50 \mathrm{AU}) \mathrm{SiO}$ and $\mathrm{H}_{2} \mathrm{O}$ but not $\mathrm{OH}$ masers can survive. For much closer orbits $(R \leq 10 \mathrm{AU})$ no maser operates. The absence of masers in the close binary systems may be explained by the tidal effects from the secondary on the maser regions. Therefore, in water-fountain sources which show $\mathrm{OH}$ and sometimes $\mathrm{SiO}$ maser activity, the orbital separation is wide enough, that the masers are not affected by the companion (Deacon et al. 2007). Alternatively, the companion may already be swept up by the primary and does not influence the maser regions which occur at much larger distances from the central star.

\section{Conclusions}

The observations show that the $\mathrm{OH}$ masers in water-fountain sources are located in aspherical shells. We performed geometrical reconstruction in order to understand the morphology of the $\mathrm{OH}$ masers. The comparison between the observations and models show that the $\mathrm{OH}$ masers of $\mathrm{W} 43 \mathrm{~A}$ are likely located in the equatorial region of the circumstellar shell, while in $\mathrm{OH}$ 12.8-0.9 the $\mathrm{OH}$ masers stem from a biconical outflow surrounding the $\mathrm{H}_{2} \mathrm{O}$ maser jet. We measured significant magnetic fields for the $\mathrm{OH}$ maser region of $\mathrm{OH} 12.8-0.9$ and $\mathrm{OH} 37.1-0.8$. This shows the possible role of the magnetic field in shaping the CSE of these stars.
Acknowledgements. This research was supported by the ESTRELA fellowship, the EU Framework 6 Marie Curie Early Stage Training program under contract number MEST-CT-2005-19669. We acknowledge MERLIN staff for their help in the observation and reducing the data. We thank Anita Richards for helping us with the initial data processing at Jodrell Bank. W.V. acknowledges support by the Deutsche Forschungsgemeinschaft through the Emmy Noether Research grant VL 61/3-1.

\section{References}

Amiri, N., Vlemmings, W., \& van Langevelde, H. J. 2010, A\&A, 509, A26 Baud, B., Habing, H. J., Matthews, H. E., \& Winnberg, A. 1979, A\&AS, 36, 193 Baud, B., Sargent, A. I., Werner, M. W., \& Bentley, A. F. 1985, ApJ, 292, 628 Blackman, E. G., Frank, A., Markiel, J. A., Thomas, J. H., \& Van Horn, H. M. 2001, Nature, 409, 485

Boboltz, D. A., \& Marvel, K. B. 2005, ApJ, 627, L45

Boboltz, D. A., \& Marvel, K. B. 2007, ApJ, 665, 680

Bowers, P. F. 1991, ApJS, 76, 1099

Chapman, J. M., \& Cohen, R. J. 1986, MNRAS, 220, 513

Claussen, M., Sahai, R., \& Morris, M. 2004, in Asymmetrical Planetary Nebulae III: Winds, Structure and the Thunderbird, ed. M. Meixner, J. H. Kastner, B. Balick, \& N. Soker, ASP Conf. Ser., 313, 331

Deacon, R. M., Chapman, J. M., Green, A. J., \& Sevenster, M. N. 2007, ApJ, 658,1096

Diamond, P. J., Norris, R. P., Rowland, P. R., Booth, R. S., \& Nyman, L. 1985, MNRAS, 212, 1

Elitzur, M. 1990, ApJ, 363, 628

Engels, D. 2002, A\&A, 388, 252

Engels, D., Schmid-Burgk, J., \& Walmsley, C. M. 1986, A\&A, 167, 129

García-Segura, G., López, J. A., \& Franco, J. 2005, ApJ, 618, 919

Habing, H. J. 1996, A\&ARv, 7, 97

Imai, H., Obara, K., Diamond, P. J., Omodaka, T., \& Sasao, T. 2002, Nature, 417, 829

Imai, H., Nakashima, J., Diamond, P. J., Miyazaki, A., \& Deguchi, S. 2005, ApJ, 622, L125

Likkel, L., Morris, M., \& Maddalena, R. J. 1992, A\&A, 256, 581

Manchado, A., Villaver, E., Stanghellini, L., \& Guerrero, M. A. 2000, in Asymmetrical Planetary Nebulae II: From Origins to Microstructures, ed. J. H. Kastner, N. Soker, \& S. Rappaport, ASP Conf. Ser., 199, 17

Modjaz, M., Moran, J. M., Kondratko, P. T., \& Greenhill, L. J. 2005, ApJ, 626, 104

Morris, M. R., Sahai, R., \& Claussen, M. 2003, in Rev. Mex. Astron. Astrof. Conf. Ser., ed. J. Arthur, \& W. J. Henney, 15, 20

Nordhaus, J., \& Blackman, E. G. 2006, MNRAS, 370, 2004

Nordhaus, J., Blackman, E. G., \& Frank, A. 2007, MNRAS, 376, 599

Nyman, L., Hall, P. J., \& Olofsson, H. 1998, A\&AS, 127, 185

Paczynski, B. 1976, in Structure and Evolution of Close Binary Systems, ed. P. Eggleton, S. Mitton, \& J. Whelan, IAU Symp., 73, 75

Reid, M. J., \& Moran, J. M. 1981, ARA\&A, 19, 231

Sahai, R., \& Trauger, J. T. 1998, AJ, 116, 1357

Sahai, R., te Lintel Hekkert, P., Morris, M., Zijlstra, A., \& Likkel, L. 1999, ApJ, 514, L115

Schwarz, H. E., Nyman, L., Seaquist, E. R., \& Ivison, R. J. 1995, A\&A, 303, 833

Sevenster, M. N. 2002, AJ, 123, 2772

Terman, J. L., \& Taam, R. E. 1996, ApJ, 458, 692

Vlemmings, W. H. T., van Langevelde, H. J., \& Diamond, P. J. 2005, A\&A, 434, 1029

Vlemmings, W. H. T., Diamond, P. J., \& Imai, H. 2006, Nature, 440, 58

Walsh, A. J., Breen, S. L., Bains, I., \& Vlemmings, W. H. T. 2009, MNRAS, 394, L70

Winnberg, A., Nguyen-Quang-Rieu, Johansson, L. E. B., \& Goss, W. M. 1975, A\&A, 38, 145

Pages 12 to 19 are available in the electronic edition of the journal at http://www . aanda. org 
A\&A 532, A149 (2011)
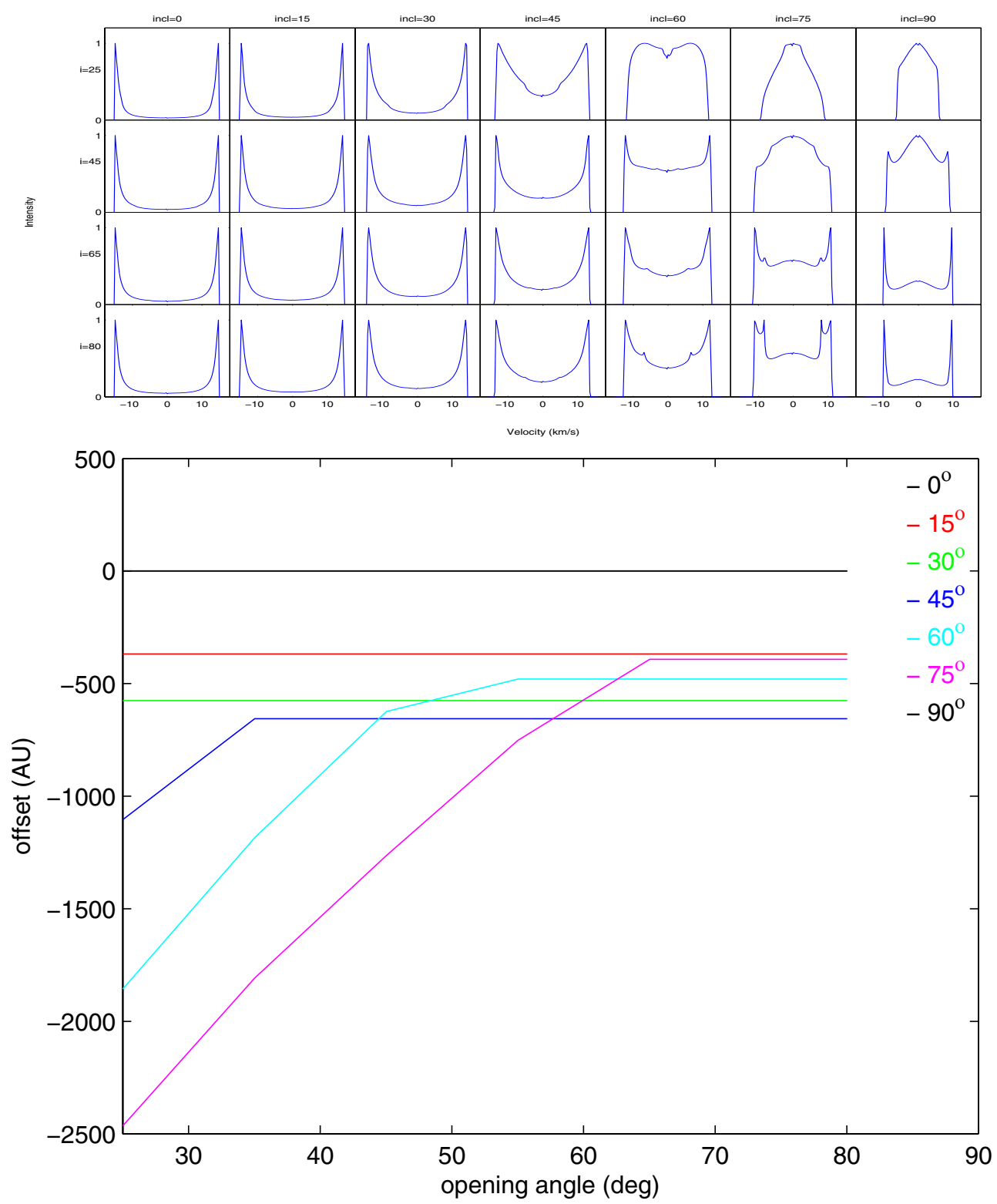

Fig. 9. As in Fig. 7, model calculations for the oblate ellipsoid $e=0.7$ with equatorial distribution, where the velocity in the equatorial region is twice that in the polar axis. 
N. Amiri et al.: The kinematics and magnetic fields in water-fountain sources based on $\mathrm{OH}$ maser observations
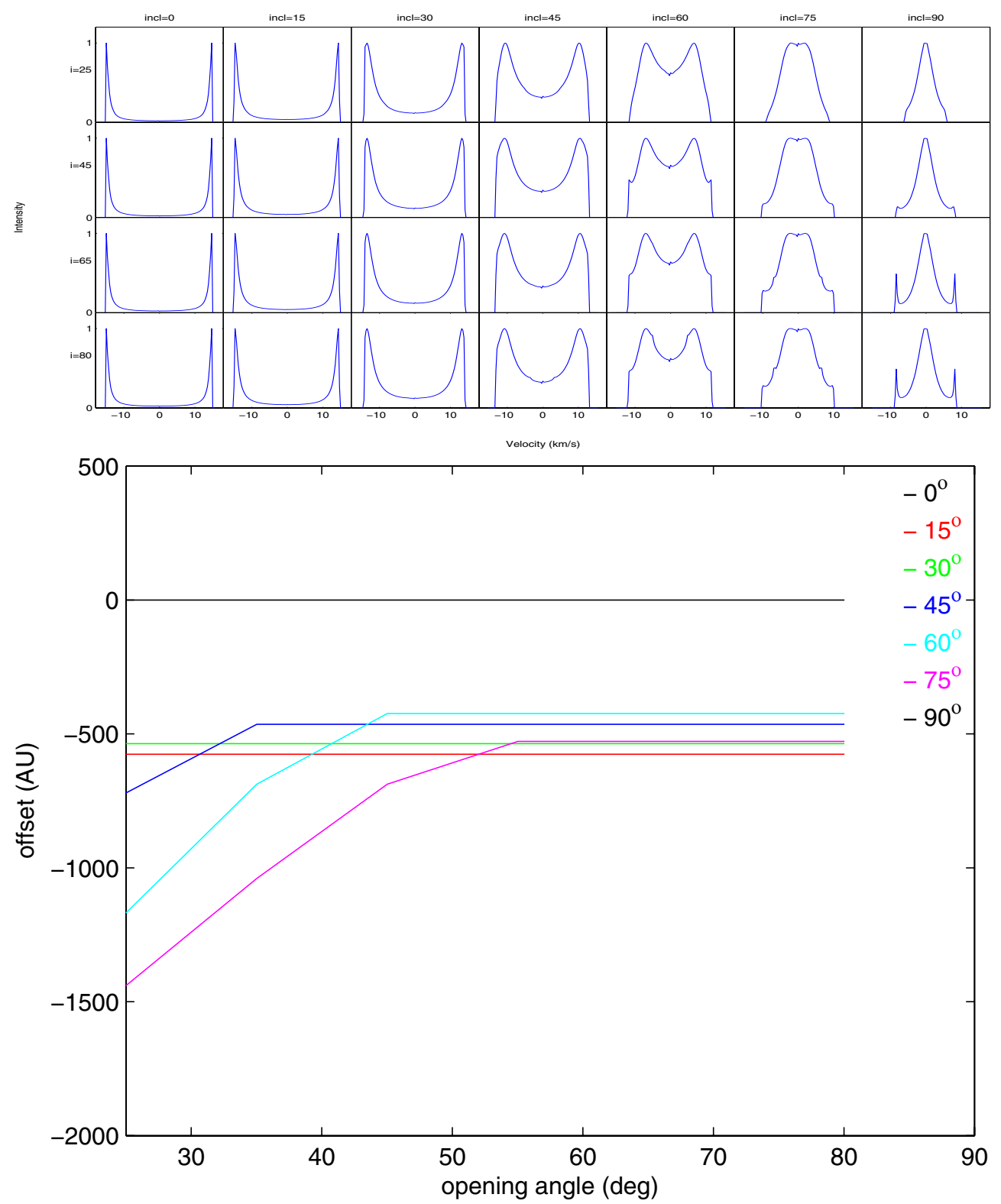

Fig. 10. As in Fig. 7, model calculations for the oblate ellipsoid $(e=0.9)$ with equatorial distribution,where the velocity in the equatorial region is four times higher than that in the polar axis. 
A\&A 532, A149 (2011)
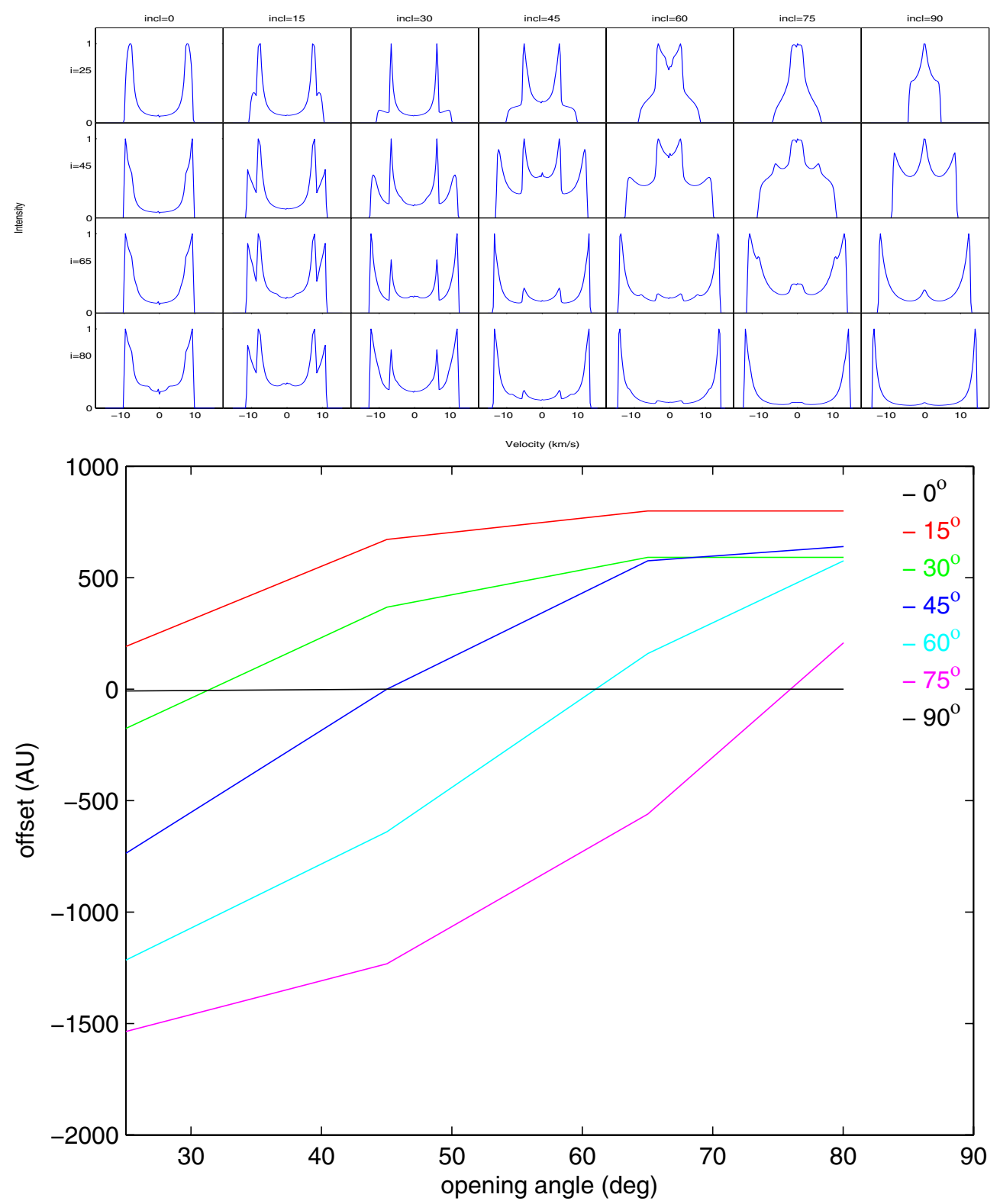

Fig. 11. As in Fig. 7, model calculations for the prolate ellipsoid $(e=0.7)$ with equatorial distribution, where the velocity in the polar axis is twice than that in the equatorial plane. 
$\mathrm{N}$. Amiri et al.: The kinematics and magnetic fields in water-fountain sources based on $\mathrm{OH}$ maser observations
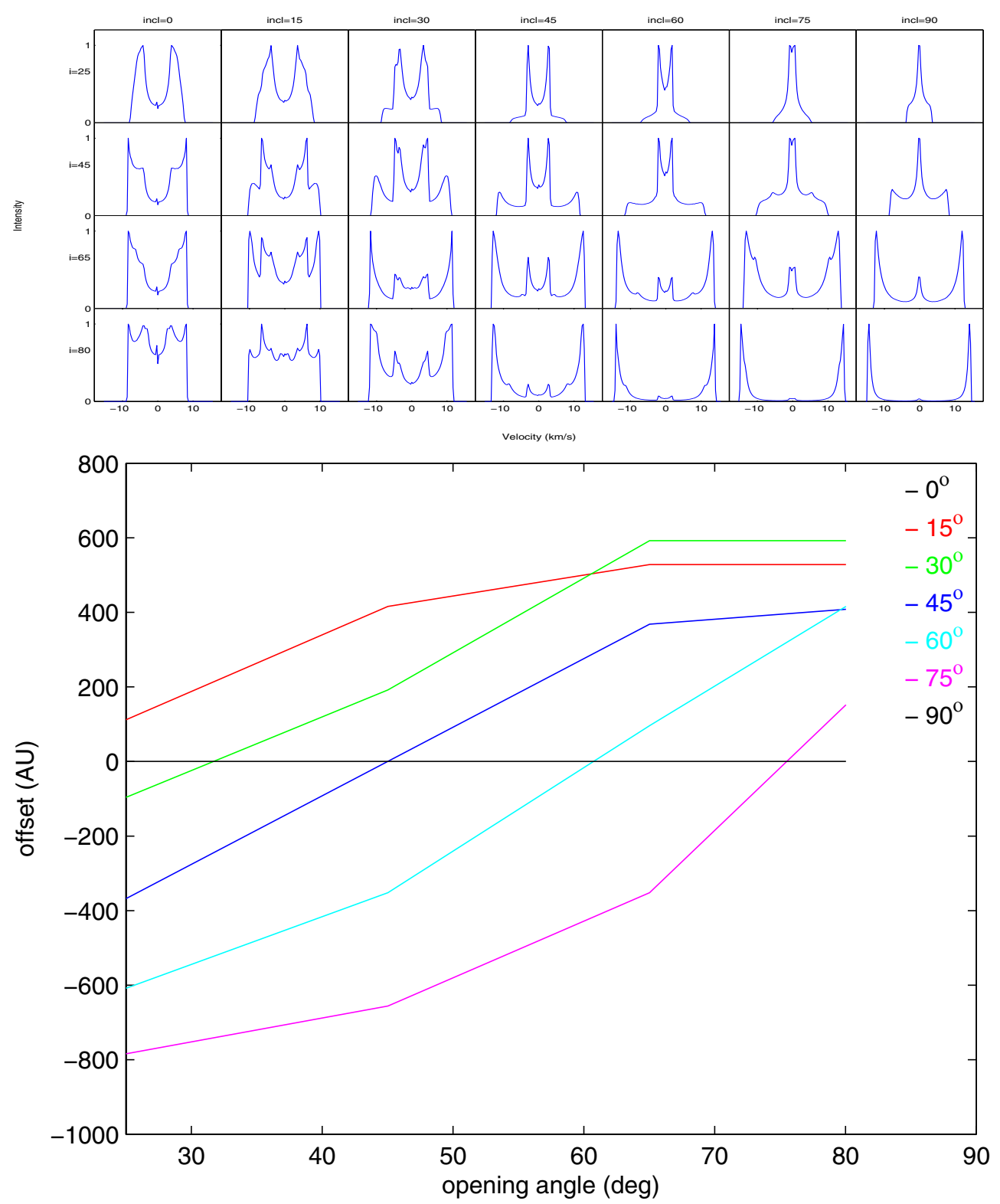

Fig. 12. As in Fig. 7, model calculations for the prolate ellipsoid $(e=0.9)$ with equatorial distribution, where the velocity in the polar axis is four times higher than that in the equatorial plane. 
A\&A 532, A149 (2011)
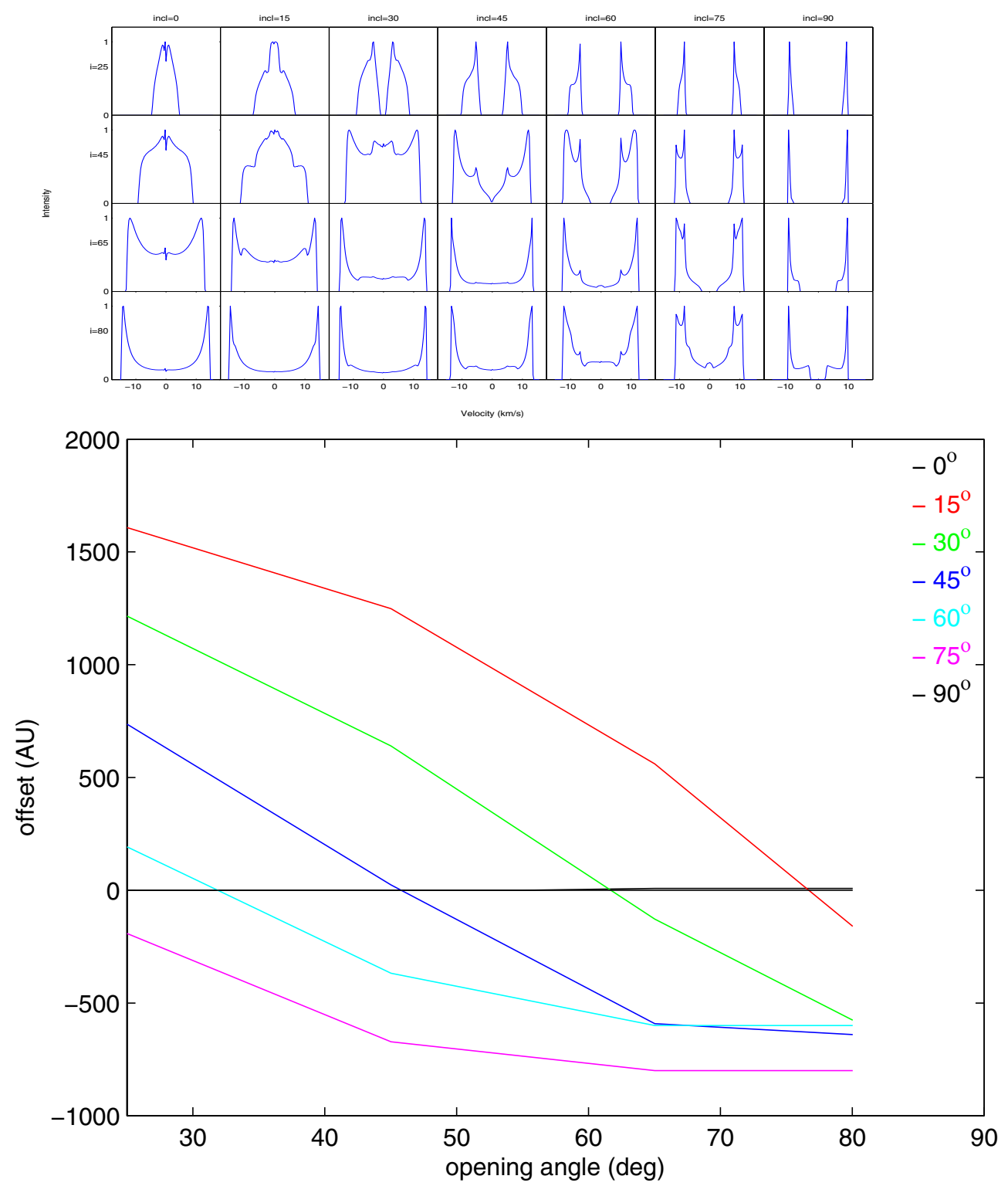

Fig. 13. As in Fig. 8, model calculations for the oblate ellipsoid $(e=0.7)$ with biconical distribution, where the outflow velocity increases towards the equatorial plane. 
N. Amiri et al.: The kinematics and magnetic fields in water-fountain sources based on $\mathrm{OH}$ maser observations
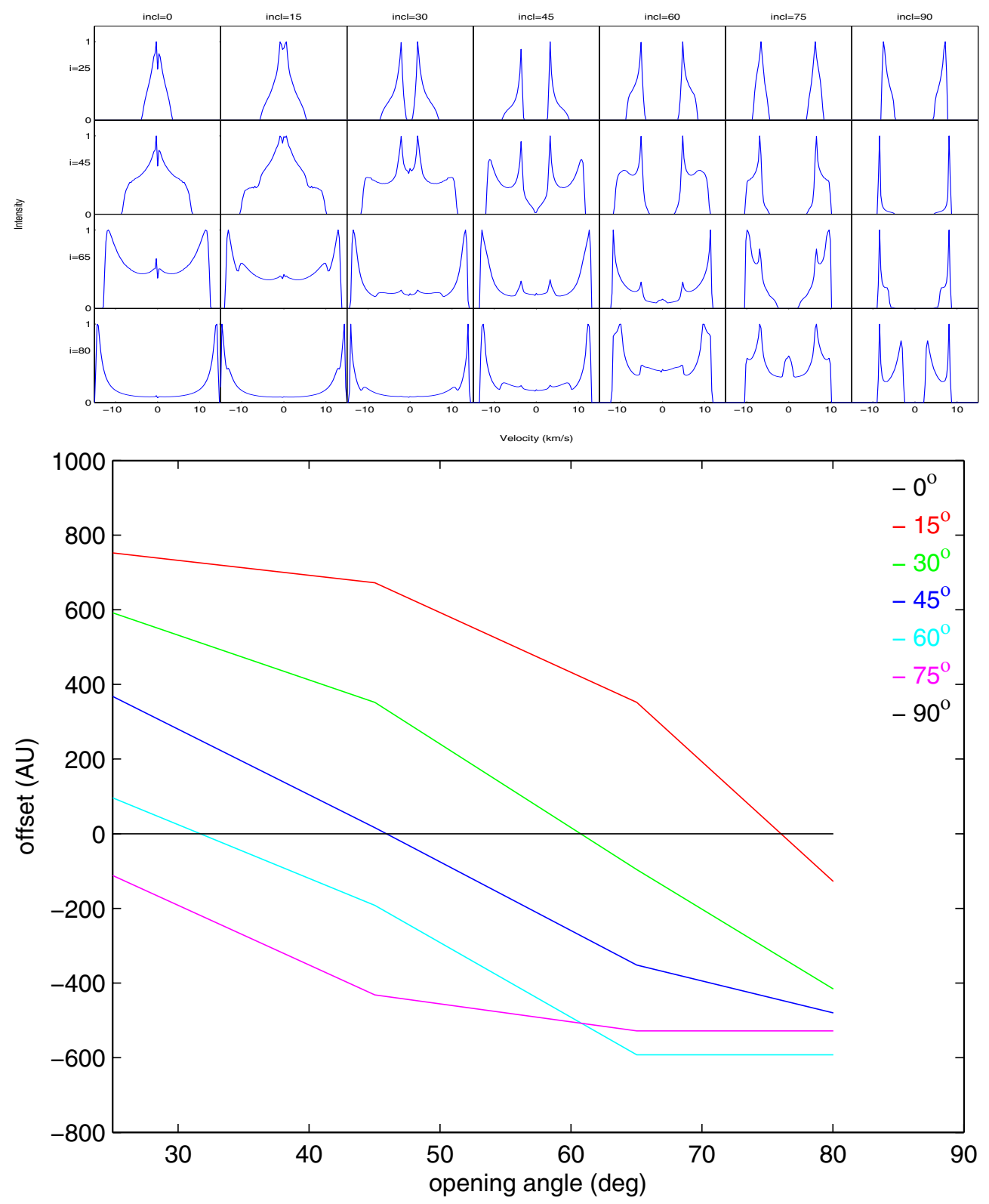

Fig. 14. As in Fig. 8, model calculations for the oblate ellipsoid $(e=0.9)$ with biconical distribution, where the outflow velocity increases towards the equatorial plane. 
A\&A 532, A149 (2011)
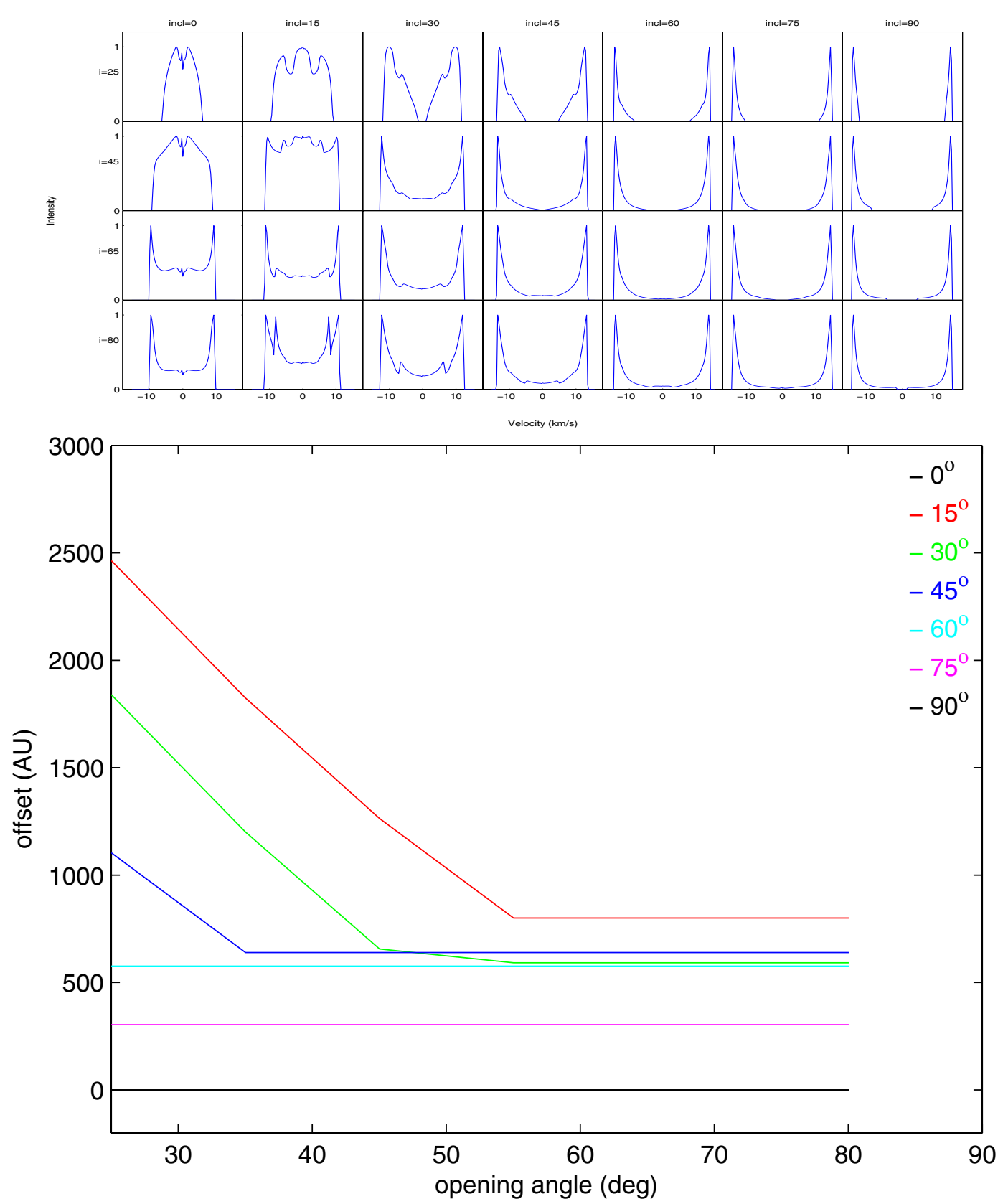

Fig. 15. As in Fig. 8, model calculations for the prolate ellipsoid $(e=0.7)$ with biconical distribution, where the outflow velocity increases towards the polar region. 
N. Amiri et al.: The kinematics and magnetic fields in water-fountain sources based on $\mathrm{OH}$ maser observations
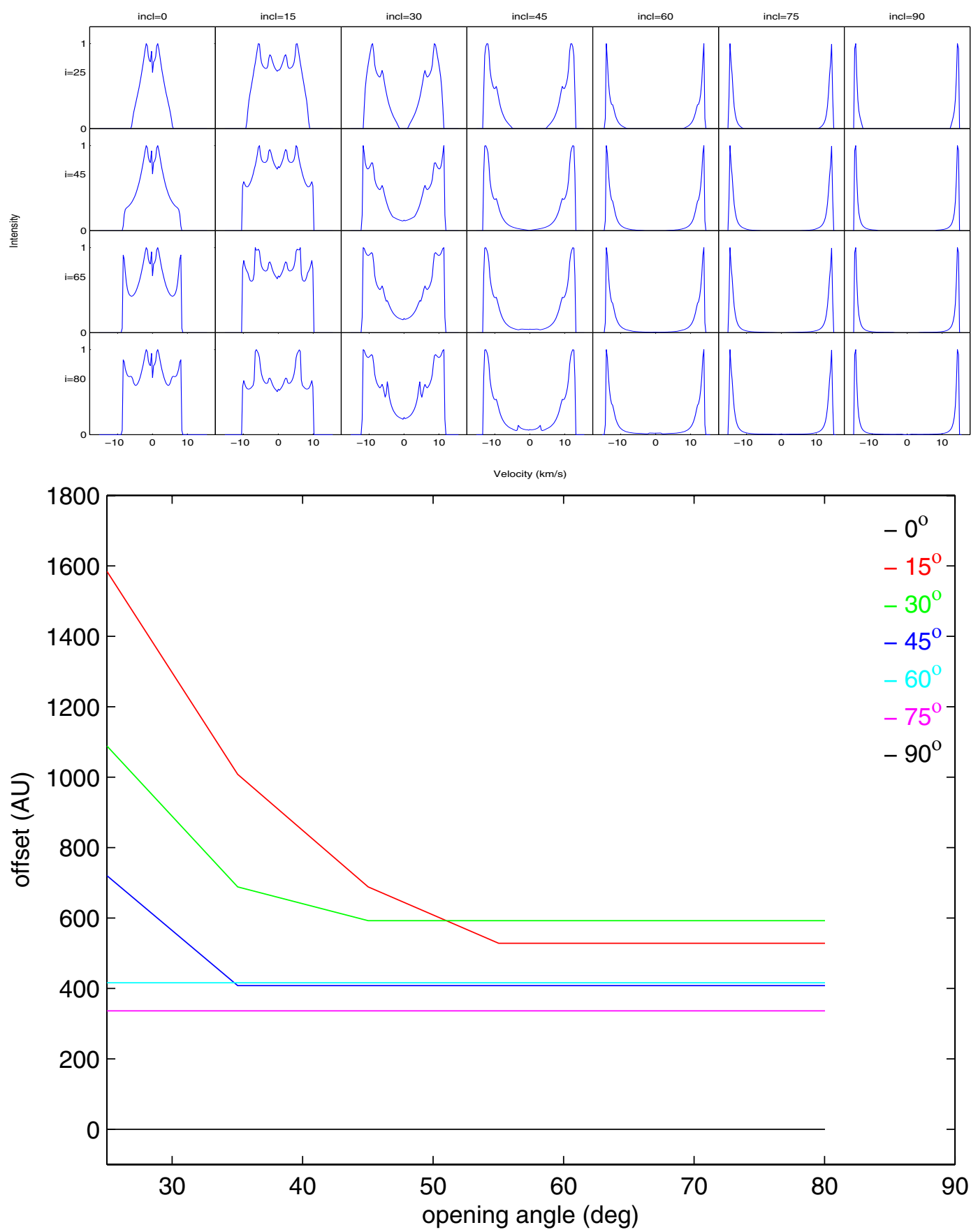

Fig. 16. As in Fig. 8, model calculations for the prolate ellipsoid $(e=0.9)$ with biconical distribution, where the outflow velocity increases towards the polar region. 